Cahiers de la recherche sur les droits

Cahiers fondamentaux Fondamentaux

Le droit de la famille en (r)évolutions

\title{
La responsabilité internationale et les droits de l'homme : le cas du Sahara occidental
}

Carlos Ruiz Miguel

\section{(2) OpenEdition}

1 Journals

Édition électronique

URL : https://journals.openedition.org/crdf/4680

DOI : $10.4000 /$ crdf. 4680

ISSN : 2264-1246

Éditeur

Presses universitaires de Caen

Édition imprimée

Date de publication : 1 novembre 2013

Pagination : 105-130

ISSN : 1634-8842

\section{Référence électronique}

Carlos Ruiz Miguel, «La responsabilité internationale et les droits de l'homme : le cas du Sahara

occidental », Cahiers de la recherche sur les droits fondamentaux [En ligne], 11 | 2013, mis en ligne le 01 décembre 2014, consulté le 14 novembre 2022. URL : http://journals.openedition.org/crdf/4680 ; DOI : https://doi.org/10.4000/crdf.4680 


\title{
La responsabilité internationale et les droits de l'homme: le cas du Sahara occidental
}

\author{
Carlos RUIZ-MIGUEL \\ Professeur de droit constitutionnel à I'Université de Saint-Jacques-de-Compostelle (Espagne)
}

I. Le conflit du Sahara occidental: des violations des droits de l'homme très longtemps négligées

II. La responsabilité de la puissance occupante:

les violations des droits de l'homme partiellement avouées par le Maroc, mais toujours impunies

III. La responsabilité de I'Espagne comme puissance administrante du Sahara occidental

IV. La responsabilité des autres États membres de la communauté internationale

V. L'Union européenne: une première dans la dénonciation des violations des droits de l'homme, mais manquant de consistance

VI. L'Union africaine et le respect des droits de l'homme au Sahara occidental: une réaction tardive, mais vigoureuse

VII.Les Nations unies: une réaction tardive et difficile face aux violations des droits de l'homme au Sahara occidental
A. L'Assemblée générale, le secrétaire général et le Conseil de sécurité
B. Les organes des Nations unies spécialisés dans le domaine des droits de l'homme
C. L'éventualité d'une Cour pénale internationale

I. Le conflit du Sahara occidental: des violations des droits de l'homme très longtemps négligées

Le conflit du Sahara occidental est une question de décolonisation. Depuis 1965, l'Assemblée générale des Nations unies s'en est saisie en la plaçant sous le cadre de la résolution 1514 sur l'octroi de l'indépendance aux peuples soumis à domination coloniale ${ }^{1}$, et, depuis 1966, cet organe a établi la formule pour procéder à ladite décolonisation: un référendum d'autodétermination des populations sahraouies autochtones ${ }^{2}$. Lorsque l'Espagne s'est finalement apprêtée à organiser le référendum d'autodétermination, l'Assemblée générale, suite à une demande du Maroc, a sollicité la Cour internationale de justice (CIJ) pour rendre un avis consultatif sur la question et demandé à l'Espagne la suspension du référendum jusqu'à ce que la CIJ publie son opinion ${ }^{3}$. L'Assemblée générale posa deux questions à la Cour: primo, si le Sahara occidental était res nullius au temps de la colonisation espagnole au $\mathrm{XIX}^{\mathrm{e}}$ siècle; et secundo, si le territoire n'était pas res nullius, quels étaient les liens qu'entretenait le territoire avec le Maroc et l'ensemble mauritanien qui étaient susceptibles 
d'affecter l'application de la résolution 1514. La Cour rendit son avis le 16 octobre 1975 en des termes clairs, affirmant que le Maroc et la Mauritanie n'avaient jamais exercé de souveraineté au Sahara occidental:

Les éléments et renseignements portés à la connaissance de la Cour montrent l'existence au moment de la colonisation espagnole de liens juridiques d'allégeance entre le sultan du Maroc et certaines des tribus vivant sur le Sahara occidental. Ils montrent également l'existence de droits, y compris certains droits relatifs à la terre, qui constituaient des liens juridiques entre l'ensemble mauritanien, au sens ou la Cour l'entend, et le territoire du Sahara occidental. En revanche, la Cour conclut que les éléments et renseignements portés à sa connaissance n'établissent l'existence d'aucun lien de souveraineté territoriale entre le territoire du Sahara occidental d'une part, le Royaume du Maroc ou l'ensemble mauritanien d'autre part. La Cour n'a donc pas constaté l'existence de liens juridiques de nature à modifier l'application de la Résolution 1514 (XV) de l'Assemblée générale des Nations Unies quant à la décolonisation du Sahara occidental, et en particulier l'application du principe d'autodétermination grâce à l'expression libre et authentique de la volonté des populations du territoire ${ }^{4}$.

En outre, la Cour reconnaît que certaines tribus ont été indépendantes:

Les renseignements dont la Cour dispose ne viennent pas étayer la prétention du Maroc selon laquelle il a exercé une souveraineté territoriale sur le Sahara occidental. Ils ne paraissent cependant pas exclure la possibilité que le Sultan ait manifesté une autorité à l'égard de certaines tribus du territoire. On ne pourrait sûrement pas le dire pour les Regueibat ou d'autres indépendants vivant sur le territoires.

Le jour même où la Cour rendit son avis, le roi du Maroc annonça son intention d'envahir le territoire pour, disait-il, «récupérer» «son» Sahara et donc la souveraineté marocaine qui, «depuis la nuit des temps», avait été «reconnue» par «tout le monde»:

Louange à Dieu, Cher peuple,

Dieu nous a enseigné que la vérité ne manque jamais de tromper.

Effectivement, cher peuple, notre droit a été reconnu et la Cour internationale de justice a répondu aux questions qu'on lui a posées.

$[\ldots]$
Les portes du Sahara nous sont juridiquement ouvertes, tout le monde a reconnu que le Sahara nous appartient depuis la nuit des temps. Il nous reste donc à occuper notre territoire ${ }^{6}$.

Le 26 octobre 1975, les forces armées du Maroc envahirent sans publicité la frontière orientale entre le Maroc et le Sahara occidental, mais l'attention publique se focalisa sur la soi-disant Marche verte composée de civils escortés par des militaires qui violèrent la frontière occidentale entre le Maroc et le Sahara occidental le 6 novembre 1975. Cette violation de la frontière fut condamnée le jour même de l'invasion par le Conseil de sécurité7.

Le 14 novembre 1975, l'Espagne (alors que Franco était déjà moribond et inconscient), le Maroc et la Mauritanie signèrent dans le secret des accords sur le Sahara occidental, les accords de Madrid. Quelques jours plus tard, après la mort de Franco le 20 novembre, un des accords fut publié ${ }^{8}$. Il établit une administration tripartite (EspagneMaroc-Mauritanie) intérimaire (jusqu'au 28 février 1976 au plus tard) pour procéder à une prétendue décolonisation du Sahara occidental sans procéder au référendum d'autodétermination exigé par les résolutions des Nations unies et avalisé par la CIJ. La présence accrue des militaires marocains (et mauritaniens) dans le territoire après cet accord déclencha la guerre du Sahara.

L'Assemblée générale se saisit dudit accord de Madrid et prononça deux résolutions refusant de cautionner cet accord: la première résolution refusait le transfert de l'administration du Sahara occidental de l'Espagne à l'entité tripartite $^{9}$; la seconde rejetait la possibilité de procéder à la décolonisation sans référendum d'autodétermination ${ }^{10}$.

Toutefois, une fois atteint le terme établi pour l'administration tripartite intérimaire, aucun référendum ne fut organisé. Le 26 février 1976, l'Espagne abandonna l'entité tripartite et déclara que l'achèvement de la décolonisation nécessitait que le peuple sahraoui se prononce valablement sur son avenir. Le lendemain, les Sahraouis, qui n'avaient pas accepté le fait accompli, sous le leadership du Front Polisario ${ }^{11}$ proclamèrent leur république, la République arabe démocratique sahraouie (RADS). Le Maroc et la Mauritanie, quelques mois plus tard (le 14 avril 1976), signèrent un traité pour diviser et répartir le territoire ${ }^{12}$.

Ces événements eurent un double effet sur la population sahraouie.

4. CIJ, «La Cour internationale de Justice rend son avis consultatif dans l'affaire du Sahara occidental», 16 octobre 1975, Recueil, 1975, p. 68, $\$ 162$.

5. Ibid., p. 48, \$105.

6. Discours du roi Hassan II, 16 octobre 1975, disponible à l'adresse: http://www.lagencedusud.gov.ma/download/discours-SM-HassanII-75-marrakech.pdf.

7. S/RES/380, 6 novembre 1975 .

8. Nations unies, «Déclaration de principes au sujet du Sahara occidental. En date du 14 novembre 1975», Recueil des traités, vol. 988,1975 , p. 258.

9. $\mathrm{A} / \mathrm{RES} / 3458 \mathrm{~A}, 19$ décembre 1975 .

10. A/RES/3458 B, 19 décembre 1975 .

11. La dénomination «Front Polisario» vient de l'acronyme espagnol du nom de l'organisation: Frente Popular para la Liberación de Saguía el Hamra y Río de Oro.

12. Nations unies, "Convention relative au tracé de la frontière d’État établie entre la République islamique de Mauritanie et le Royaume du Maroc », Recueil des traités, vol. 1035, 1977, p. 118-119 
D'un côté, une partie de la population (presque la moitié) fut obligée de s'enfuir, cherchant refuge en Algérie (seul pays limitrophe qui n'était alors pas en guerre contre le Front Polisario) et s'opposant militairement à l'invasion. La population réfugiée était essentiellement composée d'anciens, de femmes et d'enfants et subit des bombardements par l'aviation marocaine lorsqu'elle s'enfuit ${ }^{13}$. Il s'agit de la guerre du Sahara qui opposa le Front Polisario au Maroc et à la Mauritanie. Cette guerre s'acheva en 1979 lorsque les deux parties signèrent l'accord d'Alger du 5 août 1979 dans lequel la Mauritanie décida de renoncer à l'annexion du territoire ${ }^{14}$. Quand bien même, la guerre entre le Polisario et le Maroc se poursuivit jusqu'au 6 septembre 1991. Ce jour-là entra en vigueur le cessezle-feu agréé par les deux parties, prévoyant également un référendum d'autodétermination. Ces accords faisaient partie du plan de règlement accepté par les deux parties et approuvé par le Conseil de sécurité ${ }^{15}$ qui comprenait quelques autres dispositions.

De l'autre côté, l'autre partie de la population (un peu plus de la moitié) resta dans le territoire occupé ou ne fut pas capable de s'enfuir, et subit la répression des occupants, notamment du Maroc. Les souffrances de cette partie de la population sont restées inconnues ou méconnues de l'opinion publique dont l'attention était focalisée sur le développement de la guerre du Sahara. La question des droits de l'homme fut soulevée progressivement à partir de la signature et l'approbation du plan de règlement qui incluait des dispositions sur la libération des prisonniers de guerre et l'éclaircissement du sort des disparus sahraouis dans le territoire occupé ${ }^{16}$.

L'invasion et l'occupation ultérieure du territoire ont provoqué de graves violations des droits de l'homme les plus fondamentaux, voire "classiques", qui ont même été reconnues par la puissance occupante. Cependant, la «pacification» progressive du territoire a ouvert la porte à une exploitation de ses ressources naturelles et à la réalisation d'activités économiques pour attirer la présence de colons en vue de dénaturer la composition démographique du territoire. Les violations des droits économiques, sociaux et culturels constituent un autre chapitre important dans l'étude du cas du Sahara occidental, même si peu de travaux lui prêtent l'attention que cette question mérite ${ }^{17}$.

\section{La responsabilité de la puissance occupante: les violations des droits de l'homme partiellement avouées par le Maroc, mais toujours impunies}

Les obligations du Maroc en matière de droits de l'homme envers les populations sahraouies découlent de deux fondements : le droit humanitaire et le droit international des droits de l'homme.

Le droit humanitaire s'applique aux conflits armés et la présence du Maroc au Sahara occidental est le produit d'une guerre, la guerre du Sahara (1975-1991). Le statut juridique du Maroc au Sahara occidental est celui d'une puissance occupante. La présence marocaine a été qualifiée d' "occupation» par des résolutions de l'Assemblée générale des Nations unies ${ }^{18}$, ainsi que par une résolution du Parlement européen ${ }^{19}$. La doctrine la plus reconnue sur le droit de l'occupation affirme aussi que d'après ce droit le Maroc est la "puissance occupante ${ }^{20}$. Les obligations de la puissance occupante envers la population du territoire occupé sont contenues dans les articles 43-44 de la Convention concernant les lois et coutumes de la guerre sur terre conclue à La Haye le 29 juillet 1899 et dans les articles 47-78 de la Convention (IV) de Genève du 12 août 1949 relative à la protection des personnes civiles en temps de guerre, toutes deux ratifiées par le Maroc. Le Protocole additionnel aux Conventions de Genève du 12 août 1949 relatif à la protection des victimes des conflits armés internationaux (Protocole I), du 8 juin 1977, contient aussi des préceptes concernant les droits de l'homme mais cet instrument n'a été pas ratifié par le Maroc.

Le droit international des droits de l'homme est également applicable aux territoires occupés. Cette conclusion a été adoptée par la CIJ :

De manière plus générale, la Cour estime que la protection offerte par les conventions régissant les droits de l'homme ne cesse pas en cas de conflit armé, si ce n'est par l'effet de clauses dérogatoires du type de celle figurant à l'article 4 du Pacte international relatif aux droits civils et politiques. Dans les rapports entre droit international humanitaire et droits de l'homme, trois situations peuvent dès lors se présenter: certains droits peuvent relever exclusivement du droit international humanitaire; d'autres peuvent

13. Voir K. Casla, J. Garay, M. Vilches, C. Martín Beristain, J. F. Soroeta, J. M. Anda, La situación de los derechos humanos en los territorios ocupados del Sahara occidental, Vitoria, Servicio central de publicaciones del Gobierno vasco, 2008, p. 115-116.

14. A/34/427-S/13503, annexe I.

15. S/RES/658, 27 juin 1990; S/RES/690, 29 avril 1991; S/RES/725, 31 décembre 1991.

16. En septembre-octobre 1992, le Maroc déclencha une vague de répression policière et d'arrestations, peu après l'organisation par le roi Hassan II au Sahara occidental - au lieu de permettre le référendum d'autodétermination auquel il s'était engagé avec les Nations unies - d'un référendum sur une réforme de la Constitution marocaine qui avait obtenu plus de $99 \%$ de votes positifs. Voir M. de Froberville, Sahara occidental. La confiance perdue, Paris, L'Harmattan, 1996, p. 181-186; cet ouvrage est l'une des premières références bibliographiques sur la question des droits de l'homme au Sahara occidental.

17. Voir Asociación de familiares de presos y desaparecidos saharauis (AFAPREDESA), Sahara occidental, ¿ hasta cúando ? La situación de los derechos humanos en Sahara occidental, Saint-Sébastien, Tercera Prensa, 2005, p. 77-81; K. Casla et al., La situación de los derechos humanos..., p. 153-171.

18. A/RES/34/37, 21 novembre 1979; A/RES/35/19, 11 novembre 1980.

19. Résolution du Parlement européen du 27 mai 1993, JOCE, C 176, 28 juin 1993, p. 158.

20. C. Chinkin, «Laws of occupation", in Multilateralism and International Law with Western Sahara as a Case Study, N. Botha, M. Olivier, D. van Tonder (dir.), Pretoria, VerLoren van Themaat Centre - University of South Africa, 2010, p. 168-171. 
relever exclusivement des droits de l'homme; d'autres enfin peuvent relever à la fois de ces deux branches du droit international. Pour répondre à la question qui lui est posée, la Cour aura en l'espèce à prendre en considération les deux branches du droit international précitées, à savoir les droits de l'homme et, en tant que lex specialis, le droit international humanitaire ${ }^{21}$.

Les violations des droits de l'homme commises par l'État marocain ont été partiellement reconnues de manière officielle, mais restent impunies. En effet, le rapport de l'Instance Équité et Réconciliation (IER) sur les agissements des autorités marocaines entre 1956 (l'année de l'indépendance) et 1999 (le début du règne de Mohamed VI comme roi du Maroc) reconnaît beaucoup (mais pas l'ensemble) des violations des droits de l'homme commises par les autorités marocaines sur le territoire du Maroc et sur le territoire occupé du Sahara occidental, sans exiger des responsabilités ou même identifier les responsables. En revanche, n'est pas établie une reconnaissance officielle des violations commises par le Maroc après 1999 sous la conduite du présent roi, Mohamed VI.

Avant l'intronisation de Mohamed VI, le 30 juillet 1999, le Maroc avait reconnu officiellement l'existence de graves violations des droits de l'homme. C'est pendant le règne de Hassan II que se sont déroulées les dénommées "années de plomb». Mais lorsque l'on a commencé à dévoiler les preuves de ces violations (on a même évoqué un «jardin secret» du roi pour l'enterrement de certains des opposants tombés suite aux tortures), la monarchie marocaine a institué certains organes pour offrir une image plus satisfaisante du Maroc vis-à-vis des droits de l'homme. L'année 1990 a constitué à ce titre une date clé. À l'époque on savait qu'un auteur français était en train de publier un ouvrage sur les violations des droits de l'homme dans plusieurs centres secrets de détention, ouvrage qui aura un impact très fort sur les relations franco-marocaines. L'ouvrage en question, de Gilles Perrault, est composé des témoignages de certains rescapés des prisons secrètes marocaines et, notamment, de Malika Oufkir ${ }^{22}$. Face à ces révélations, le roi décida d'une stratégie préventive et approuva une mesure, plutôt cosmétique: la création d'un soi-disant Conseil consultatif des droits de l'homme ${ }^{23}$.
L'«Exposé des motifs» du dahir contient plusieurs considérations qui méritent d'être reproduites:

Les droits de l'homme ont été sans cesse pris en considération $[\ldots]$.

L'application de la législation en vigueur, l'action des institutions représentatives, les recours offerts par l'appareil judiciaire ont largement assuré la réalisation de cet idéal.

Cependant, comme le prouve l'exemple de bien d'autres pays, les moyens ainsi mis en œuvre au service des droits de l'homme peuvent à la suite d'abus ou d'erreurs inhérents à la nature humaine ou par suite des lacunes juridiques non encore comblées, ne pas être suffisants pour assurer une réalisation parfaite des buts poursuivis ${ }^{24}$.

Ledit Conseil, cependant, n'a que des compétences consultatives auprès du roi, mais «à la majorité des deux tiers le Conseil peut se saisir de sa propre initiative des questions sur lesquelles il estime utile d'informer $»$ le roi ${ }^{25}$.

Après la mort de Hassan II le 24 juillet 1999, le nouveau roi essaie de donner une nouvelle image pour se différencier de celle de son père. En l'an 2001, il décide ainsi de réformer le Conseil consultatif des droits de l'homme ${ }^{26}$ pour élargir un peu ses compétences, mais sans lui attribuer une quelconque compétence décisionnelle, de sorte que le Conseil reste essentiellement le même. On pourrait dire que plus qu'une "mutation» du Conseil, la nouvelle régulation développe la structure préalablement existante.

Le dahir de 2001 persiste dans la négation de la responsabilité de l'État concernant les violations graves des droits de l'homme. C'est ainsi que l' «Exposé des motifs » renvoie au dahir de 1990 quand il dit:

En prévision des lacunes qui pourraient affecter les textes juridiques, et des abus éventuels, auxquels pourrait donner lieu l'exercice de l'autorité et qui demeurent inhérents à la nature humaine même lorsqu'elle est exercée en toute bonne foi ${ }^{27}$.

Mais, même avec toutes ces préventions, on établit, de façon expresse, la compétence pour

[...] examiner, de sa propre initiative ou sur requête de la partie concernée, les cas de violations des droits de l'homme qui lui sont soumis et faire les recommandations qui s'imposent à l'autorité compétente ${ }^{28}$.

21. CIJ, «Conséquences juridiques de l'édification d'un mur dans le territoire palestinien occupé», Recueil des arrêts, avis consultatifs et ordonnances, 2004, p. 178, \$106.

22. G. Perrault, Notre ami le roi, Paris, Gallimard, 1990. Le livre est paru le 12 septembre 1990, mais il est clair que Hassan II savait que le livre était en cours de préparation / publication. La source principale du livre est le témoignage de Malika Oufkir, fille du colonel Oufkir, qui selon Perrault fut assassiné par Hassan II lui-même après le coup d'État fomenté contre lui en 1972. Après ce coup d'État, toute la famille Oufkir, la veuve et tous les enfants (certains étant encore alors de jeunes enfants) furent emprisonnés dans de terribles conditions dans un bagne secret. En 1987, Malika et une de ses sœurs réussirent à s'enfuir mais toutes les deux furent découvertes. Elles ont alors été détenues en résidence surveillée. Malika Oufkir elle-même a raconté son expérience: M. Oufkir, La prisonnière, récit recueilli et présenté par M. Fitoussi, Paris, B. Grasset, 1999.

23. Dahir nº 1-90-12 du 20 avril 1990 relatif au Conseil consultatif des droits de l'homme, Bulletin officiel du Royaume du Maroc, $\mathrm{n}^{\circ} 4044,2$ mai 1990.

24. Ibid., "Exposé des motifs».

25. Ibid., art. 6.2.

26. Dahir n 1-00-350 du 10 avril 2001 portant réorganisation du Conseil consultatif des droits de l'homme, Bulletin officiel du Royaume du Maroc, $n^{\circ}$ 4926, 16 août 2001. Il est alors surprenant de lire un chercheur de l'Université de Rabat traiter de ce texte en ces termes (malgré les propos répétés de Belguendouz le dahir a été publié le 16 août 2001, pas le 10 avril 2001): «Dahir nº 1.00.350 publié au B.O. le 10 avril 2001 et portant réorganisation du $\mathrm{CCDH}$ »; A. Belguendouz, Le Conseil de la communauté marocaine à l'étranger. Une nouvelle institution en débat, Fiesole, Institut européen de Florence - Robert Schuman Centre for Advanced Studies, 2009, p. 7, n. 15 et p. 30.

27. Ibid., «Exposé des motifs».

28. Ibid., art. 2 . 
Malgré cette modification de la norme relative au Conseil consultatif, il sera rapidement évident que la réforme était tout à fait insuffisante pour satisfaire aux demandes de justice des victimes des graves violations des droits de l'homme au Maroc et dans les territoires occupés du Sahara occidental.

La nécessité de donner une légitimation au royaume de Mohamed VI poussera ce dernier à faire un pas en avant. Dans son discours du 7 janvier 2004, le roi annonça une nouvelle initiative visant à régler la question des violations des droits de l'homme, tout en limitant la possibilité de porter des accusations sur les violations commises dans la période de son propre règne. La création de l'institution annoncée en janvier 2004 ne sera concrétisée qu'en avril $2004^{29}$.

Le nouveau dahir (publié officiellement, on doit insister sur ce point, seulement en arabe) introduit un changement profond parce que, pour la première fois, on reconnaît qu'il y a eu des « violations graves des droits de l'homme » ${ }^{30}$ notamment des «disparitions forcées» et des «détentions arbitraires». Mais le dahir introduit une autre nouveauté importante en reconnaissant des «responsabilités de l'État ${ }^{31}$ dans ces violations.

L'Instance Équité et Réconciliation (IER) rédigea un rapport final fin novembre 2005 contenant des recommandations adressées au roi. Cependant, le texte du rapport final n'existe qu'en arabe ${ }^{32}$ (tout comme le dahir instituant l'IER) et l'on dispose en français seulement d'un résumé ${ }^{33}$. Le rapport reconnaît, parmi d'autres violations, que dans la période examinée,

[1]'analyse des dossiers soumis à l'Instance, ainsi que les témoignages oraux lors des auditions publiques ou à huis clos, ont révélé le recours systématique à la torture [...].

L'IER, en phase avec la prétention officielle d'annexion du Sahara occidental, ne fait apparemment pas une analyse différenciée des situations du Maroc et du Sahara occidental, mais la pratique de la recherche avait ellemême montré la différence. En fait, l'IER a organisé des auditions publiques des victimes des graves violations des droits de l'homme au Maroc. La huitième et dernière audition publique, prévue à El-Aaiun, la capitale du Sahara occidental, fut reportée avant d'être finalement annulée, apparemment en raison des troubles politiques qui secouèrent le territoire en mai 2005. Dans le cadre de ses travaux de recueil et d'enregistrement des témoignages, l'IER a également enregistré et transcrit le témoignage de Sahraouis ayant de hautes responsabilités dans l'administration marocaine. Un de ces responsables, Khalihenna Ould Errachid, président du Conseil royal consultatif pour les affaires sahariennes, a révélé de gravissimes violations des droits de l'homme par le Maroc. Sa déclaration est restée secrète jusqu'au moment où elle fut révélée et publiée par un journal arabophone marocain en $2008^{34}$.

Le rapport de l'IER a été critiqué à plusieurs titres. La première critique a émané de Human Rights Watch qui a publié un rapport ${ }^{35}$ clarifiant certaines des données contenues dans le rapport de l'IER, dont le fait que la plupart des disparus enregistrés par l'IER étaient originaires du Sahara occidental ${ }^{36}$. La deuxième critique est venue d'Amnesty International qui a remarqué que le mandat de l'IER «ne couvrait pas toutes les atteintes aux droits humains commises entre 1956 et 1999 », et que «malheureusement - malgré l'indignation des victimes et des organisations de défense des droits humains l'identification des auteurs des graves violations en avait été exclue ${ }^{37}$. La troisième critique, conforme à la méthodologie employée par l'IER, est venue d'un expert dans l'enquête sur les violations des droits de l'homme dans plusieurs situations internationales, Carlos Beristain, qui a publié une recherche exhaustive sur le Sahara occidental ${ }^{38}$. Celui-ci a pointé quelques insuffisances, parmi d'autres, dans le rapport de l'IER: existence de graves inexactitudes factuelles ${ }^{39}$; manque important de garanties pour les victimes qui n'étaient pas protégées contre d'éventuelles persécutions pendant les audiences de l'IER ${ }^{40}$; absence d'une liste des cas contenant les circonstances basiques des affaires et les institutions responsables, contrairement

29. Dahir n 1-04-42 du 10 avril 2004 portant approbation du statut de l'Instance Équité et Réconciliation, Bulletin officiel du Royaume du Maroc, $\mathrm{n}^{\circ}$ 5203, 12 avril 2004. Il faut noter que ce dahir est publié seulement en arabe, le bulletin officiel no 5203 n'ayant pas été traduit en français Il existe une traduction française du dahir faite par l'IER dans sa page web, qui précise cependant qu'il s'agit d'une traduction non officielle.

30. Ibid., «Exposé des motifs »; art. 9.1.

31. Ibid., art. 9.5.

32. Texte disponible à l'adresse: $h t t p: / / w w w . i e r . m a / r u b r i q u e . p h p 3 ? i d \_r u b r i q u e=316$.

33. Texte disponible à l'adresse : http://www.ier.ma/article.php3?id_article=1496.

34. Voir M. de Froberville, Sahara occidental. Le droit à l'indépendance, Alger, Éditions ANEP, 2009, p. 254-255: «"Il y a des gens [...], il s'agit de trois ou quatre officiers de l'armée, qui ont commis ce qu'on peut appeler des crimes de guerre contre des prisonniers hors du contexte de la guerre" [...] "de nombreux civils ont été jetés dans le vide par hélicoptère ou enterrés vivants" pour le seul fait d'être sahraouis».

35. Human Rights Watch, "La Commission marocaine de vérité. Le devoir de mémoire honoré à une époque incertaine», vol. 17, $\mathrm{n}^{\circ}$ 11(E), 2005, disponible à l'adresse: http://www.hrw.org/sites/default/files/reports/morocco1105frwcover.pdf.

36. Ibid., p. 40 .

37. Amnesty International, «Des promesses non tenues. L'Instance Équité et Réconciliation et le suivi de ses travaux», Amnesty International Publications, MDE 29/oo1/2010, disponible à l'adresse: http://www.amnesty.org/ar/library/asset/MDE29/o01/2010/ar/668448ac-f45d-4ee9-a72ae48ea8182872/mde29001201ofra.html.

38. C. Martín Beristain, E. González Hidalgo, El oasis de la memoria: memoria histórica y violaciones de derechos humanos en el Sáhara Occidental, Bilbao, Hegoa, 2012, 3 vol. Cette publication peut être téléchargée gratuitement à l'adresse: http://publicaciones.hegoa.ehu.es/publications/281. Il s'agit, à mon avis, de la recherche la plus exhaustive menée jusqu'à présent sur le sujet.

39. Ibid., vol. II, p. 273-275.

40. Ibid., vol. II, p. 275-278. 
à la pratique des «commissions vérité» dans d'autres pays, et absence d'une recherche approfondie des affaires les plus emblématiques ${ }^{41}$.

Le dahir instituant l'IER introduit une surprenante disposition qui de facto évite les possibilités d'enquêter et, donc, d'indemniser les violations des droits de l'homme commises après la montée au pouvoir de Mohamed VI. L'article 9.4 du dahir permet d'indemniser les préjudices matériels et moraux subis par les victimes ou leurs ayants droit si les demandes ont été "soumises à l'Instance précitée après expiration du délai fixé auparavant à fin décembre 1999" ou "dans le délai d'un mois nouvellement fixé du 12 janvier 2004 au vendredi 13 février 2004 ». Mais, comment peut-on introduire une demande entre le 12 janvier 2004 et le 13 février $2004 \ldots$ si le dahir instituant l'Instance Équité et Réconciliation est adopté le 10 avril? Il est tout à fait clair que cette disposition absurde essaie de cacher le fait que l'IER est une institution créée pour juger le royaume du père de Mohamed VI, mais tout en négligeant les violations des droits de l'homme commises dans son royaume. Une des critiques adressées par Human Rights Watch à l'IER est qu'elle ne peut enquêter sur les violations des droits de l'homme commises après 1999 qui ont atteint spécialement trois groupes de personnes: les Sahraouis, les islamistes et les opposants laïcs:

Bien que l'IER soit une preuve évidente des progrès réalisés en matière de droits humains, elle a fonctionné au cours d'une période où ces acquis avaient enregistré des reculs: la répression des présumés islamistes et des militants de l'indépendance du Sahara occidental, la poursuite persistante des journalistes et l'utilisation de la force pour disperser des manifestations pacifiques ${ }^{42}$.

Le Conseil consultatif des droits de l'homme du Maroc a publié en décembre 2009 un rapport sur le suivi des recommandations de l'Instance Équité et Réconciliation. Ce rapport fait état de ce que le Conseil a accompli des recommandations concernant quatre axes: la recherche de la vérité, la réparation individuelle, la réparation communautaire, les réformes institutionnelles et juridiques. À cette date, ledit Conseil a publié trois des annexes au rapport de suivi de 2009. Toutes les trois (annexe 1 sur les cas des disparitions forcées, annexe 2 sur la réparation individuelle et annexe 3 sur le bilan du Conseil dans le domaine des réformes juridiques et institutionnelles) sont publiées seulement en arabe, même si le français est aussi une langue officielle du Maroc. Comme d'habitude, à chaque fois que le Maroc décide de publier une information officielle «délicate» il la publie seulement en arabe pour limiter la possibilité de connaissance de ce type d'informations dans les pays occidentaux. L'annexe $1^{43}$ reconnaît que les responsables des graves violations sont des membres de l'armée, de la gendarmerie et des forces auxiliaires, même si le document omet de préciser nombre de détails qui sont forcément connus par les auteurs du rapport: on occulte les lieux d'enterrement de plusieurs victimes, on ne diffuse pas l'identité des personnes tombées au combat - même si le rapport reconnaît avoir ces données - ou on diffuse des données incomplètes sur l'enlèvement ou la détention de plusieurs cas. L'annexe omet l'identification des responsables de graves violations des droits de l'homme.

La situation des droits de l'homme au Sahara occidental est actuellement toujours très grave après l'accession au trône de Mohamed VI. Amnesty International a déclaré qu'après la publication de son rapport plusieurs des violations rapportées par l'IER jusqu'en 1999 continuent à se produire ${ }^{44}$. Beristain dans son ouvrage a fait état de ces violations commises après 1999, jusqu'à la fin de sa recherche en $2012{ }^{45}$. Cependant, le travail de Beristain et Hidalgo, qui ont mené une recherche exhaustive sur les violations des droits de l'homme, ne fait pas état des violations liées à la méconnaissance du droit à un procès équitable. En effet, il existe une relation inversement proportionnelle entre la diminution des situations de disparitions forcées (qui sont presque terminées) et l'augmentation des violations du droit à un procès équitable, justement parce que les victimes qui ne sont pas l'objet de disparitions forcées sont soumises à des procédures incompatibles avec le respect des droits de l'homme ${ }^{46}$. Le procès le plus médiatisé des prisonniers sahraouis, celui des prisonniers d'Akdeim Izik, en est la preuve. $\mathrm{Si}$, certes, on trouve un rapport qui affirme que le procès a été «équitable» ${ }^{47}$, il n'est pas moins certain que des institutions internationales de protection des droits de l'homme ${ }^{48}$ ainsi que des organisations non gouvernementales possédant une longue tradition dans

41. C. Martín Beristain, E. González Hidalgo, El oasis de la memoria ..., vol. II, p. 284-295.

42. Human Rights Watch, «La Commission marocaine de vérité...», p. 48.

43. Texte disponible à l'adresse: http://www.ccdh.org.ma/spip.php?article4268.

44. Amnesty International, «Des promesses non tenues...».

45. C. Martín Beristain, E. González Hidalgo, El oasis de la memoria..., vol. I, p. 383 sq

46. Voir K. Casla et al., La situación de los derechos humanos..., p. 148-153; Swedish Section of the International Commission of Jurists, Trial Observations in Western Sahara. Reports and Legal Analysis, Stockholm, Swedish Section of the International Commission of Jurists, 2011, p. 99 sq.

47. «Rapport des observateurs français membres de l'Association de Promotion des Libertés Fondamentales sur le procès des personnes poursuivies à raison de leur participation aux événements criminels de Gdeim Izik près de Laayoune, tenu à Rabat (Maroc) du $1^{\text {er }}$ février au 17 février 2013 ", disponible à l'adresse: http://www.europe-maroc.com/wp-content/uploads/GDEIM-IZIK-Le-rapport-des-observateurs-Fr1.pdf. Le rapport est signé par Christophe Boutin, Jean-Yves de Cara, Mathieu Cardon, Sylvie Ceccaldi-Guebel, Ariane Guignot, Michel de Guillenchmidt, Thierry Rambaud, Charles Saint-Prot et Joanna Schmidt-Szalewski. Il faut néanmoins observer que selon une publication française, ce rapport a été commandé par un organisme officiel marocain, le Conseil national des droits de l'homme, créé par Mohamed VI en 2011. Voir «Le procès Gdeim Izik fêté à Paris ", Maghreb confidentiel, no 1054, 28 février 2013, http://www.africaintelligence.fr/MC-/cercles-de-pouvoir/2013/o2/28/ le-proces-gdeim-izik-fete-a-paris,107946952-ART.

48. Rupert Colville, porte-parole du Haut-Commissariat des Nations unies aux droits de l'homme, «Press Briefing Notes on Egypt and Western Sahara », 19 février 2013, disponible à l'adresse: http://www.ohchr.org/EN/NewsEvents/Pages/DisplayNews.aspx?NewsID=13010\&LangID=E ; 
le domaine des droits de l'homme ${ }^{49}$ ont déclaré qu'il s'agissait d'un cas présentant de nombreuses violations du droit à un procès équitable.

Si la responsabilité des violations graves des droits de l'homme commises au Sahara occidental est partiellement reconnue, il demeure exact que ces violations restent toujours impunies, hormis une exception: depuis le début de l'invasion du Sahara occidental une seule violation des droits de l'homme a été punie... et la sanction a été presque vidée de sa substance. Le 30 octobre 2005 , quelques mois après le déclenchement de l'intifada sahraoui le 21 mai 2005, le jeune Hamdi Lembarki a succombé aux blessures provoquées par les matraques de deux policiers marocains lorsqu'il exerçait son droit à manifester pacifiquement à El-Aaiun. Le 20 juin 2007, les deux policiers marocains, Abderrahim Emssaued et Hassan Rochdi, ont été condamnés à dix ans de prison pour ce décès. Mais le 4 mars 2008, ils ont été libérés par le tribunal d'El-Aaiun après avoir purgé seulement deux des dix ans requis dans le verdict de condamnation ${ }^{50}$.

Toutes les autres violations des droits de l'homme restent impunies. Le cas le plus étonnant est celui des événements d'Akdeim Izik où plusieurs Sahraouis ont été condamnés sans aucune preuve pour de prétendus assassinats de plusieurs policiers dont le nom n'est pas mentionné dans le verdict de condamnation, alors que les assassinats de plusieurs Sahraouis bien identifiés (Najam el Garhi, Khadijetou Ebhaïa, Baby Hamday) lors de ces événements restent impunis.

\section{La responsabilité de l'Espagne comme puissance administrante du Sahara occidental}

L'Assemblée générale des Nations unies a qualifié l'Espagne de «puissance administrante» du Sahara occidental depuis $1965^{51}$. Ce statut est maintenu par l'Assemblée générale même après la signature des accords de Madrid (instituant une prétendue administration intérimaire tripartite) du 14 novembre de $1975^{52}$. L'Espagne a abandonné le territoire (l'espace terrestre) du Sahara occidental le 26 février 1976 , mais les Nations unies considèrent encore l'Espagne comme puissance administrante du territoire ${ }^{53}$. Il faut noter que même si l'Espagne ne possède plus l'espace terrestre, elle exerce encore quelques compétences sur l'espace maritime et aérien du territoire ${ }^{54}$. Les obligations des puissances administrantes des territoires non autonomes sont de caractère politique et socio-économique. Ces deux types d'obligations ont des effets sur les droits de l'homme. Politiquement, d'après l'article 73 de la Charte des Nations unies,

[1] es Membres des Nations Unies qui ont ou qui assument la responsabilité d'administrer des territoires dont les populations ne s'administrent pas encore complètement elles-mêmes reconnaissent le principe de la primauté des intérêts des habitants de ces territoires.

L'article 73 ajoute dans son alinéa a) que la puissance administrante a une obligation

[...] d'assurer, en respectant la culture des populations en question, leur progrès politique, économique et social, ainsi que le développement de leur instruction, de les traiter avec équité et de les protéger contre les abus ${ }^{55}$.

Le même article dans son alinéa b) établit l'obligation

[...] de développer leur capacité de s'administrer ellesmêmes, de tenir compte des aspirations politiques des populations et de les aider dans le développement progressif de leurs libres institutions politiques.

Et dans son alinéa c) affirme aussi la nécessité « d'affermir la paix et la sécurité internationales». La "primauté des intérêts des habitants », la «protection contre les abus» et l' "affirmation de la paix et la sécurité internationales", doivent indéniablement être interprétées aujourd'hui comme une référence au respect des droits de l'homme.

L'Espagne peut alors exercer sa juridiction sur le territoire non autonome qui est encore sous sa responsabilité. Devant l'Audiencia Nacional (le parquet espagnol) ont été introduites deux plaintes de natures opposées.

Le 14 décembre 2006, le citoyen sahraoui Ali Omar Buzeid ${ }^{56}$ et d'autres ont porté plainte contre plusieurs responsables marocains ${ }^{57}$ pour génocide au Sahara occidental de 1976 à 1987. Il faut cependant noter que la plainte était

Commission africaine des droits de l'homme et des peuples, «Déclaration de la commission africaine des droits de l'homme et des peuples sur le procès et la condamnation de vingt-cinq civils sahraouis par un tribunal militaire marocain», 23 février 2013, disponible à l'adresse: http:// www.achpr.org/fr/press/2013/02/d142.

49. Human Rights Watch, «Maroc: le procès des civils sahraouis a été entaché d'irrégularités », $1^{\text {er }}$ avril 2013, disponible à l'adresse : https://www.hrw. $\mathrm{org} / \mathrm{fr} / \mathrm{news} / 2013 / 04 / 01 / \mathrm{maroc}-\mathrm{le}$-proces-des-civils-sahraouis-ete-entache-d-irregularites; Amnesty International, «Maroc et Sahara occidental : des Sahraouis condamnés doivent être rejugés de manière équitable devant des tribunaux civils», 18 février 2013, disponible à l'adresse: http:// www.amnesty.org/fr/news/morocco-convicted-sahrawis-must-receive-fair-trial-2013-02-18.

50. Voir Département d'État des États-Unis, Country Reports on Human Rights Practices 2008, Washington, Joint Committee Print, 2010, vol. I, p. 2010 et 2027.

51. A/RES/2072, 16 décembre 1965 .

52. A/RES/3458-A, 10 décembre 1975

53. Nations unies, lettre datée du 29 janvier 2002, adressée au président du Conseil de sécurité par le secrétaire général adjoint aux affaires juridiques et conseiller juridique, document $\mathrm{S} / 2002 / 161, \S 6$.

54. Voir C. Ruiz-Miguel, «Spain's Legal Obligations as Administering Power of Western Sahara», in Multilateralism and International Law..., p. 207.

55. Nous soulignons.

56. Ali Omar Buzeid avait publié son témoignage auparavant (en 2005) dans AFAPREDESA, Sahara occidental..., p. $104-108$.

57. Parmi les responsables accusés on trouve quatre généraux (Abdelaziz Bennani, Hosni Bensliman, Abdelhak El Kadiri et Hamidou Lanigri), le haut responsable des prisons (Abdelhafid Ben Hachem) et l'actuel haut responsable des services secrets extérieurs (Yassine Mansouri). 
fondée sur la compétence «universelle» alors reconnue dans la loi espagnole et non sur le fait que le territoire demeure sous la responsabilité administrante espagnole. Le juge d'instruction de l'Audiencia Nacional a déclaré cette plainte irrecevable dans une décision du 29 octobre $2007^{58}$. La procédure a été longtemps retardée par le juge d'instruction, mais reprise lors du changement de juge d'instruction. Le 13 mars 2013, le juge a entendu le témoignage d'Aminatou Haïdar, la défenseure des droits de l'homme et ancienne disparue.

Seulement deux mois après la déclaration de recevabilité de la plainte sahraouie contre des hauts responsables marocains le 26 décembre 2007 , l'Association sahraouie de défense des droits de l'homme et trois particuliers ont porté plainte devant l'Audiencia Nacional espagnole contre des hauts responsables du Front Polisario. Le juge d'instruction de l'Audiencia Nacional a déclaré la plainte recevable dans une décision du 16 août $2012^{59}$, sans pour autant encore entendre un quelconque témoignage concernant cette plainte.

Malheureusement, il semble fort peu probable que ces procès puissent aboutir. Comme l'a souligné un procureur devant le Tribunal Supremo (Cour de Cassation espagnole), le Royaume du Maroc ignore les demandes de collaboration judiciaire émanant des cours espagnoles lorsque les accusés sont des autorités civiles et militaires marocaines. En plus, puisque l'Espagne ne reconnaît pas la République arabe sahraoui démocratique (RASD), il est également très difficile d'adresser des demandes de collaboration judiciaire aux cours et autorités sahraouis ${ }^{60}$. La situation peut ressembler à l'enquête menée par un juge français sur la disparition à Paris de l'opposant marocain Mehdi Ben Barka en 1965, encore ouverte, mais bloquée faute de collaboration des autorités marocaines et de diligence des autorités françaises.

\section{La responsabilité des autres États membres de la communauté internationale}

Même s'il existe des organisations et traités internationaux à vocation universaliste, le système du droit international public n'est pas totalement centralisé. Sa particularité réside en un respect des règles qui incombe en premier lieu aux États membres de la communauté internationale.
Mais cette responsabilité est conditionnée par deux circonstances. Primo, s'il s'agit de faire respecter les droits de l'homme il est tout à fait clair que cette tâche ne peut être remplie que si l'État qui exige ce respect en démontre luimême une application. Secundo, le poids de la censure ou la pression exercée par un État dépend du poids politique ou économique de celui-ci. Certains États possèdent les deux (comme les États-Unis), mais d'autres États ont, dans le domaine des droits de l'homme, un poids politique beaucoup plus fort que leur poids économique (les pays scandinaves, par exemple).

On peut dire que les États-Unis d'Amérique sont les plus actifs dans la défense des droits de l'homme au Sahara occidental, avec une action qui s'est déployée en trois directions : la législation, l'élaboration des rapports et la pression diplomatique.

Le Congrès américain a adopté une loi en décembre 2011 pour soumettre les aides financières des États-Unis vers d'autres pays à la présentation préalable d'un rapport sur le respect des droits de l'homme par le pays concerné. Ce texte prévoit que pour donner suite au financement militaire américain au profit du Royaume du Maroc, le titulaire du Département d'État doit présenter au Comité des assignations un rapport renseignant sur les efforts effectués par le gouvernement du Maroc pour « respecter les droits des individus à exprimer pacifiquement leurs opinions concernant le statut et le futur du Sahara occidental et documenter les violations des droits de l'homme» et «faciliter l'accès sans restrictions au Sahara occidental des organisations des droits de l'homme, journalistes et représentants des gouvernements étrangers ${ }^{61}$.

Le Département d'État nord-américain fait un examen annuel des divers pays du monde concernant leur respect des droits de l'homme, qui est ensuite publié dans deux sortes de rapports: d'un côté, on peut trouver les rapports par pays sur la situation des droits de l'homme dans leur ensemble; et d'un autre côté, les rapports spécifiques sur le respect de la liberté religieuse. Le Sahara occidental fait l'objet de rapports spécifiques, différenciés de ceux sur le Maroc dans ce domaine. Les rapports sur la situation générale des droits de l'homme au Sahara occidental montrent que les violations des droits de l'homme dans les territoires occupés par le Maroc sont persistantes ${ }^{62}$.

En plus de la publication des rapports, les États-Unis exercent des pressions diplomatiques discrètes qui ont été révélées par Wikileaks. Dans le cas du Sahara occidental,

58. Auto [ordonnance] du 29 octobre 2007 du juge d'instruction $n^{\circ} 5$ de la Audiencia Nacional, Diligencias Previas [enquête preliminaire]. PROC. ABREVIADO 362 /2007-A.

59. Auto [ordonnance] du 16 août 2012 du juge d'instruction n ${ }^{\circ} 5$ de la Audiencia Nacional, Diligencias Previas [enquête preliminaire]. PROC. ABREVIADO $1 / 2008$-A.

60. Voir E. Fungairiño, «Justicia española en el Sahara Occidental», La Razón, 10 mai 2013.

61. Consolidated Appropriations Act, 2012, sec. 7041, disponible à l'adresse: http://www.gpo.gov/fdsys/pkg/BILLS-112hr2055enr/pdf/BILLS-112hr2055enr. pdf: " (g) MOROCCO. - Prior to the obligation of funds appropriated by this Act under the heading "Foreign Military Financing Program" for assistance for Morocco, the Secretary of State shall submit a report to the Committees on Appropriations on steps being taken by the Government of Morocco to - (1) respect the right of individuals to peacefully express their opinions regarding the status and future of the Western Sahara and to document violations of human rights; and (2) provide unimpeded access to human rights organizations, journalists, and representatives of foreign government to the Western Sahara» (nous traduisons).

62. Le dernier rapport publié correspond à 2011, il est disponible à l'adresse : http://www.state.gov/j/drl/rls/hrrpt/humanrightsreport/index. htm?dlid=186441. 
un des documents publiés montre clairement que la légation nord-américaine a exercé des pressions sur le Maroc pour obtenir une amélioration de la situation des droits de l'homme dans le territoire occupé du Sahara occidental ${ }^{63}$.

D’autres pays ont également exercé des pressions en faveur du respect des droits de l'homme au Sahara occidental. Ces actions sont de deux natures. Quelques actions sont menées au moyen de la diplomatie «parlementaire» et d'autres de la diplomatie "classique».

Dans le cadre de la diplomatie «parlementaire», c'est probablement le Sénat du Mexique qui est le Parlement le plus engagé dans la défense des droits de l'homme au Sahara occidental. On peut trouver plusieurs résolutions parlementaires adressées au gouvernement marocain à ce sujet. Cette chambre s'est montrée particulièrement active dans la défense des droits de l'homme au Sahara occidental, demandant l'attribution à la Mission des Nations unies pour l'organisation d'un référendum au Sahara occidental (MINURSO) de compétences dans le domaine des droits de l'homme (2012), dénonçant la destruction du camp Akdeim Izik (2010) ou exigeant la fin de la déportation de la défenseure des droits de l'homme et ancienne disparue, Aminatou Haïdar (2009) ${ }^{64}$. D'autres Parlements ont également montré leur préoccupation face aux développements survenus concernant la situation des droits de l'homme au Sahara occidental, tels que les Parlements de la Suède $(2008)^{65}$ ou de l'Afrique du Sud $(2013)^{66}$.

Dans le cadre de la diplomatie «classique» certaines chancelleries ont manifesté leur préoccupation sur la situation des droits de l'homme au Sahara occidental (Australie $\left.{ }^{67}\right)$ et ont même mené des discussions bilatérales ou multilatérales sur le sujet avec le gouvernement marocain. C'est le cas du Danemark ${ }^{68}$ ou de l'Irlande ${ }^{69}$.

\section{L'Union européenne: une première dans la dénonciation des violations des droits de l'homme, mais manquant de consistance}

Au sein de l'Union européenne, le Parlement européen a été le premier à dénoncer les violations des droits humains, même avant l'approbation du plan de règlement. Cependant, la position de l'Union européenne, et du Parlement européen en l'espèce, manque parfois de cohérence, premièrement parce qu'elle ne tire pas les conséquences de sa dénonciation de la violation des droits de l'homme dits «classiques», mais aussi parce qu'elle a négligé longtemps les droits dits « économiques » qui ont également été violés au Sahara occidental.

Le Parlement européen est probablement le premier organe international à dénoncer les violations des droits de l'homme au Sahara occidental, puisqu'il l'a fait depuis 1987 . On a suggéré plusieurs explications à cet égard ${ }^{70}$. Primo, l'Acte unique européen, entré en vigueur en juillet 1987, octroyait quelques compétences sur les affaires extérieures au Parlement européen ${ }^{71}$. Secundo, la présence au Parlement européen, des députés espagnols, à ce moment les plus engagés pour la cause sahraouie et qui étaient présents depuis l'adhésion de l'Espagne aux Communautés européennes le $1^{\text {er }}$ janvier 1986; ces députés avaient promu la création de l'intergroupe «Paix

63. Voir, par exemple, le câble daté du 8 juillet 2009, disponible à l'adresse: http://wikileaks.org/cable/2009/07/09RABAT584.html.

64. «Punto de acuerdo por el que se exhorta al Consejo de Seguridad de la Organización de las Naciones Unidas a ampliar las atribuciones de la Misión de Naciones Unidas para el Referéndum del Sahara occidental en la protección de los derechos humanos del pueblo saharaui», 29 mars 2012, disponible à l'adresse: http://www.senado.gob.mx/index.php?ver=sp\&mn=3\&sm=3\&id=31459; «Punto de acuerdo por el que el Senado de la República expresa al gobierno de la República Árabe Saharaui Democrática, su más profundo pesar y enérgica indignación por los actos en los que perdieron la vida ciudadanos saharauis el pasado 8 de noviembre en El Aaiún, y manifiesta su más sentido pésame a las familias de las víctimas », 16 novembre 2010, disponible à l'adresse: http://www.senado.gob.mx/index.php?ver=sp\&mn=2\&sm=2\&id=27655; «Punto de acuerdo por el que el Senado de la República reprueba la detención de la defensora de los derechos humanos del pueblo saharaui Aminatou Haidar», 8 décembre 2009, disponible à l'adresse: $h$ ttp://www.senado.gob. $\mathrm{mx} /$ index.php? $\mathrm{ver}=\mathrm{sp} \& \mathrm{mn}=2 \& \mathrm{sm}=2 \& \mathrm{id}=23426$.

65. Voir en ligne: http://www.riksdagen.se/sv/Dokument-Lagar/Utskottens-dokument/Betankanden/2007o8Manskliga-rattigheter-i_ GVo1UUg/?html=true.

66. Voir «Human rights situation in Western Sahara - Presentation to the Portfolio Committee on International Relations and Cooperation - Deputy Minister Ebrahim ", 20 mars 2013, disponible à l'adresse: http://www.safpi.org/sites/default/files/publications/dirco_western_sahara.pdf.

67. Réponse de M. Downer, ministre des Affaires étrangères australien, 18 septembre 2007, disponible à l'adresse: http://parlinfo.aph.gov.au/ parlInfo/search/display/display.w3p;db=CHAMBER;id=chamber\%2Fhansardr\%2F2007-09-18\%2Foo94;query=Id\%3Achamber\%2Fhansardr ${ }_{22} \mathrm{~F}_{2007-09-18 \% 2 \mathrm{Foooo} .}$

68. Déclaration du gouvernement danois devant le Parlement, 22 novembre 2010, disponible à l'adresse: http://www.ft.dk/samling/20101/almdel/ euu/spm/63/svar/768249/938266/index.htm?.

69. Parmi l'ensemble des déclarations, voir les déclarations du Vice-Premier ministre et ministre des Affaires étrangères et du Commerce irlandais (Deputy Eamon Gilmor): Written Answers no 15-23, Human Rights Issues, 20 février 2013, disponible à l'adresse: http://oireachtasdebates. oireachtas.ie/Debates\%20Authoring/DebatesWebPack.nsf/takes/dail2013022000070; Written Answers, Human Rights Issues, 18 juillet 2012 , disponible à l'adresse: http://oireachtasdebates.oireachtas.ie/Debates\%20Authoring/DebatesWebPack.nsf/takes/dail2012071800056?opendocum ent; Written Answers, Human Rights Issues, 22 mai 2012, disponible à l'adresse: http://oireachtasdebates.oireachtas.ie/Debates\%2oAuthoring/ DebatesWebPack.nsf/takes/dail2012052200035?opendocument.

70. Voir Á. Urruela, «El Parlamento Europeo frente al conflicto del Sahara Occidental», Papers, no 46, 1995, p. 111, 113.

71. Selon l'article 30.4 de l'Acte unique européen, JOCE, L 169, 29 juin 1987, p. 1 sq.: «Les Hautes Parties contractantes assurent l'association étroite du Parlement européen à la coopération politique européenne. À cette fin, la présidence informe régulièrement le Parlement européen des thèmes de politique étrangère examinés dans le cadre de la coopération politique et elle veille à ce que les vues du Parlement européen soient dûment prises en considération dans ces travaux». 
pour le peuple sahraoui ». Mais ses résolutions n'ont pas été caractérisées par la continuité et on peut différencier plusieurs étapes.

La période la plus avant-gardiste et la plus engagée pour les droits de l'homme au Sahara occidental s'établit entre 1987 et 1995.

Avant 1987, le Parlement européen n'a produit qu'une seule résolution sur le Sahara occidental, évoquant seulement la question de la guerre et du processus politique pour régler le conflit, et prônant une approche voisine des positions officielles du Maroc ${ }^{72}$.

La résolution du 14 mai $1987^{73}$ traite du «sort de la famille du général Oufkir et des centaines des disparus au Maroc», mais on y trouve aussi une référence aux disparus sahraouis puisque le Parlement européen réclame «des informations sur les centaines de disparus marocains et sahraouis».

La résolution du 17 décembre $1987^{74}$ se fait l'écho d'une vague de répression déclenchée par l'État marocain contre des civils sahraouis qui avaient manifesté pacifiquement, lorsque l'ONU et l'Organisation de l'unité africaine (OUA) avaient décidé d'envoyer une mission technique au Sahara occidental dans le cadre de leurs efforts pour appliquer les résolutions pertinentes des Nations unies sur le Sahara occidental. Le Parlement européen mentionne la détention de «dizaines» d'hommes et de femmes sahraouis par les forces policières marocaines et exprime le «risque de torture» que subissent les détenus. Dans cette résolution, le Parlement européen proteste contre ces détentions de personnes qui manifestaient pacifiquement et insiste pour la libération immédiate des détenus et le respect de leurs droits. Malheureusement, l'appel du Parlement européen n'a eu aucun écho auprès des gouvernements et, lors de cette vague de répression déclenchée en 1987, beaucoup de Sahraouis ont été portés «disparus » jusqu'en 1991, ont subi des tortures et quelques-uns ont été assassinés.

L'année suivante, le Parlement européen a approuvé une nouvelle résolution, du 15 mars 1989, mais qui ne se focalise que sur la question politique et n'insiste plus sur les droits de l'homme dits «classiques». Le Parlement européen reconnaît que la crise doit être résolue en application «du droit inaliénable du peuple sahraoui à l'autodétermination et à l'indépendance ${ }^{75}$.

La quatrième résolution du Parlement européen sur le Sahara occidental, du 15 février $1990^{76}$, reprend la ligne de la résolution de décembre 1987, et dénonce les violations des droits de l'homme dits «classiques». Cette résolution proteste contre la vague de répression de 1987 qui avait provoqué "plusieurs centaines d'arrestations en novembre 1987" parmi lesquels des hommes, femmes et enfants, dont le sort demeure inconnu. Cette résolution souligne, elle aussi, l'absence d'information sur quatrevingts «disparus » depuis 1976 dans le territoire occupé.

Quelques mois plus tard, le 11 octobre 1990, le Parlement européen approuve une nouvelle résolution ${ }^{77}$ qui traite du droit humanitaire, et notamment des droits des prisonniers de guerre. À cet égard, le Parlement européen dénonce le fait que le Maroc ne veut pas rapatrier deux cents soldats marocains libérés par le Front Polisario et refuse de donner des renseignements sur les prisonniers de guerre sahraouis.

L'année suivante, 1991, est celle de l'approbation du plan de règlement par le Conseil de sécurité qui a donné beaucoup d'espérances. Cette année-là, le Parlement européen a adopté deux résolutions sur le Sahara occidental, en relation avec le plan de règlement et négligeant la question des droits de l'homme dits «classiques». La résolution du 18 avril $1991^{78}$ ne contient pas d'éléments spécifiques sur la question des droits de l'homme, mais la résolution du 12 septembre $1991^{79}$ inclut un paragraphe qui accuse le Maroc de violer la liberté de conscience lorsque cet État conditionne la libération des prisonniers de guerre sahraouis à ce qu'ils «reconnaissent» que le Sahara occidental «fait partie intégrante du Maroc».

En 1992, le régime marocain déclenche une nouvelle vague de répression contre les populations sahraouies des territoires occupés. Dans ce contexte, le Parlement européen adopte sa résolution du 29 octobre $1992^{80}$ qui dénonce la sanglante répression du droit à manifester pacifiquement dans plusieurs villes du Sahara occidental et les emprisonnements et disparitions qui ont suivi.

Le référendum d'autodétermination prévu dans le plan de règlement approuvé par le Conseil de sécurité a été fixé pour la fin de l'année 1992, mais le Maroc a réussi à le reporter, ce qui a donné lieu à des protestations des Sahraouis dans les territoires occupés. Ce contexte explique la résolution du 27 mai $1993^{81}$ qui est l'une des plus énergiques dénonciations des violations des droits de l'homme perpétrées au Sahara occidental par le régime marocain. Cette résolution rappelle que depuis 1976 plus de mille personnes ont été portées «disparues» dans les territoires occupés du Sahara occidental. La résolution

[...] condamne les arrestations arbitraires, la pratique de la "disparition», de la torture et de la détention au

72. Voir notamment la résolution du 12 mars 1981, JOCE, C 77, 6 avril 1981, p. 43-45

73. JOCE, C 156, 15 juin 1987, p. 145-146. Ce même jour le Parlement européen avait approuvé une autre résolution «sur les droits de l'homme au Maroc» (JOCE, C 156, 15 juin 1987, p. 145).

74. JOCE, C 13, 18 janvier 1988, p. 102.

75. JOCE, C 96, 17 avril 1989, p. 59-61.

76. JOCE, C 68, 19 mars 1990, p. 143-144.

77. JOCE, C 284, 12 novembre 1990, p. 131-132.

78. JOCE, C 129, 20 mai 1991, p. 125-126.

79. JOCE, C 267, 12 septembre 1991, p. 129-130.

80. JOCE, C 305, 23 novembre 1992, p. 153.

81. JOCE, C 176, 28 juin 1993, p. 158. 
secret ainsi que toutes les formes de violation des droits de l'homme [...], demande aux autorités communautaires et aux États membres de tout mettre en œuvre pour assurer la protection de la population sahraouie [...], renouvelle sa demande à la coopération politique européenne pour exiger du Maroc le respect des droits de l'homme et des accords internationaux, notamment la Déclaration universelle des droits de l'homme des Nations unies et les Conventions de Genève [...], demande au Maroc la libération des Sahraouis détenus et des personnes emprisonnées en raison de leur activité politique ou syndicale [...], renouvelle son appel au gouvernement marocain pour qu'il autorise l'accès immédiat aux territoires occupés du Sahara occidental d'observateurs internationaux, de missions d'organisations humanitaires et de défense des droits de l'homme.

On doit souligner que le Parlement européen a qualifié expressément les territoires sahraouis sous contrôle marocain de "territoires occupés».

De la même manière, on trouve la résolution du 10 février $1994^{82}$ qui non seulement montre la préoccupation du Parlement européen face aux «tentatives permanentes du gouvernement marocain visant à empêcher l'application du processus de paix des Nations unies au Sahara occidental» mais réitère en même temps sa condamnation des violations des droits de l'homme déjà présente dans la résolution du 27 mai 1993. En outre, devant la persistance des violations des droits de l'homme, il

[...] demande aux autorités de l'Union et aux gouvernements des États membres, dans le cadre de leurs relations politiques, économiques et commerciales avec le Maroc de tout mettre en ouvre pour contraindre le gouvernement marocain à mettre un terme au système des "disparitions " et à appliquer le plan de paix des Nations unies au Sahara occidental.

La résolution du Parlement européen du 16 mars $1995^{83}$ sur le Sahara occidental accorde une attention particulière au blocage du plan de règlement des Nations unies pour le Sahara occidental mais rappelle aussi sa demande adressée au gouvernement marocain de «ne plus interdire l'accès au Sahara occidental des observateurs étrangers, et notamment des parlementaires, juristes et journalistes » et réitère son appel aux autorités de l'Union européenne et aux gouvernements des États membres à faire pression sur le Maroc pour que cet État respecte ses engagements internationaux.

Suite aux condamnations à de lourdes peines de certains étudiants sahraouis qui manifestaient à El-Aaiun le 11 mai 1995, le Parlement européen a adopté sa résolution du 13 juillet $1995^{84}$, réclamant la libération immédiate des étudiants emprisonnés et invitant le gouvernement marocain «à respecter les conventions internationales qu'il a souscrites en matière de droits de l'homme».

La deuxième étape caractérisant les résolutions du Parlement européen sur le Sahara occidental comprend les années 1995-2005, phase qui correspond au processus de négociation d'un accord d'association entre l'Union européenne et le Maroc. Durant cette période, on constate un assouplissement de la pression exercée par le Parlement européen puisque ses résolutions soit évoquent seulement les développements du règlement du conflit aux Nations unies, soit parlent des droits de l'homme «classiques » en utilisant un langage très nuancé.

En 1996 on procède à la signature entre l'Union européenne et le Maroc de l'accord d'association de 1996. La même année, le 6 juin 1996, le Parlement européen approuve deux résolutions qui semblent être en quelque sorte "compensatoires». La première est une résolution législative où le Parlement européen donne son avis conforme à la conclusion de l'accord euro-méditerranéen d'association avec le Maroc qui accorde des avantages commerciaux importants au Maroc ${ }^{85}$. La seconde est une résolution «sur la situation des droits de l'homme au Maroc» (sic) ${ }^{86}$ qui traite, malgré son titre, de la situation des droits de l'homme aussi bien au Maroc qu'au Sahara occidental et parle également du blocage politique du plan de règlement approuvé par l'ONU.

Pourtant, le 10 mars $1998^{87}$, le Parlement européen n'adopte pas une résolution sur le Sahara occidental, mais une recommandation qui ne fait que référence au processus de paix onusien et non aux droits de l'homme. L'année suivante, on approuve une résolution du 14 janvier $1999^{88}$ qui ne parle également que du processus de paix de l'ONU.

La résolution du 16 mars $2000^{89}$ évoque aussi cette question, mais contient aussi quelques références aux violations des droits de l'homme. Cependant, le langage reste très nuancé et ne mentionne plus l'appel à faire pression sur le Maroc. Ainsi, on «demande la garantie d'une liberté totale de manifestation pour les habitants du Sahara occidental» et on "réitère la décision d'envoyer au Sahara occidental une mission qui serait chargée d'évaluer l'évolution de la situation sur le terrain".

On doit attendre trois ans pour voir une nouvelle référence au Sahara occidental dans une résolution du Parlement européen. Et celle-ci se trouve dans un paragraphe d'une résolution consacrée aux relations méditerranéennes de l'Union européenne. La résolution relative à Euromed du 20 novembre $2003^{90}$ contient en effet un

82. JOCE, C 61, 28 février 1994, p. 177.

83. JOCE, C 89, 10 avril 1995, p. 159.

84. JOCE, C 249, 25 septembre 1995, p. 159-160.

85. JOCE, C 81, 24 juin 1996, p. 15.

86. Ibid., p. 15-16.

87. JOCE, C 104, 6 avril 1998, p. 29-30.

88. JOCE, C 104, 14 avril 1999, p. 112-113.

89. JOCE, $\mathrm{C} 377,29$ décembre 200o, p. 354-355.

90. JOCE, C $87 \mathrm{E}, 7$ avril 2004, p. 500-502. 
bref paragraphe ( $\left.\mathrm{n}^{\circ} 22\right)$ demandant l'application du plan de paix des Nations unies. La résolution du 14 avril $2005^{91}$ ne parle plus ni des droits de l'homme ni du règlement du conflit, mais se borne à traiter de l'aide humanitaire aux réfugiés sahraouis à Tindouf. La résolution suivante qui fait référence au Sahara occidental est celle du 28 avril $2005^{92}$ sur les droits de l'homme dans le monde et la politique de l'Union européenne. Cette résolution contient un paragraphe où l'on dit que le Parlement européen

[...] prend note, avec préoccupation, des informations faisant état des violations des Droits de l'homme au Sahara occidental, y compris en matière de liberté de parole et liberté de circulation, et encourage le Maroc et le Front Polisario à progresser sur la base du plan Baker, reconnu au niveau international; invite le Maroc et le Front Polisario à libérer tous les prisonniers de guerre.

La troisième période que l'on peut discerner pourrait s'étendre de fin 2005 jusqu'à aujourd'hui. Durant cette période, le Parlement européen prête moins d'attention au processus politique devant l'ONU, bloqué depuis la renonciation de James Baker comme envoyé personnel du secrétaire général de l'ONU en juin 2004 à attirer l'attention une nouvelle fois sur les droits de l'homme. On doit noter qu'au cours de cette période se sont passés quelques événements très importants dans les territoires occupés du Sahara occidental qui expliquent le durcissement de la position du Parlement européen : l'intifada de 2005 , la déportation de la défenseure des droits de l'homme sahraouie Aminatou Haïdar en 2009 et la destruction du camp de protestation à Akdeim Izik en 2010.

Le point d'inflexion est provoqué par le déclenchement de l'intifada sahraoui le 21 mai 2005 suivi par une sanglante répression du régime marocain et par des détentions arbitraires, des procès non équitables et des emprisonnements pour des motifs politiques. La résolution du Parlement européen du 27 octobre $2005^{93}$ constitue la réponse à cette nouvelle phase. Elle rappelle la question des plus de cinq cents «disparus » et demande la libération des détenus et prisonniers après l'intifada de mai 2005. On peut cependant relever quelques éléments nouveaux dans la résolution, notamment la référence à la question des «ressources naturelles énergétiques». En outre, la résolution insiste sur la nécessité d'envoyer une mission pour enquêter sur la situation au Sahara occidental.

En novembre 2009, le régime marocain décide la déportation d'Aminatou Haïdar, ancienne disparue (entre 1987 et 1991) et emprisonnée suite à l'intifada (entre 2005 et 2006). $\mathrm{M}^{\mathrm{me}}$ Haïdar entame une grève de la faim en signe de protestation et soulève des soutiens croissants en Espagne et en Europe. Le Parlement européen se dispose à voter une importante résolution dont le texte a été préparé le 16 décembre $2009^{94}$. Elle constitue un désaveu complet de la politique du Maroc au Sahara occidental occupé et le régime marocain décide d'autoriser le retour de $\mathrm{M}^{\mathrm{me}}$ Haïdar dans son pays, le Sahara occidental, pour éviter que la résolution du Parlement européen ne soit votée.

En novembre 2011, on assiste aux plus graves événements socio-politiques au Sahara occidental depuis l'occupation marocaine. Plus de vingt mille Sahraouis sortent de la capitale du Sahara occidental, El-Aaiun, et décident d'installer un camp de protestation contre la discrimination politique, économique et sociale qu'ils subissent face aux colonisateurs (un modèle de protestation suivi ensuite dans d'autres pays arabes comme le Bahrein, l'Égypte ou la Palestine). Le régime marocain écrase la protestation avec la destruction totale du camp le 8 novembre 2010, ce qui conduit le Parlement européen à adopter une résolution le 25 novembre $2010^{95}$. Cette résolution contient quelques éléments nouveaux. Le plus important est l'exigence de ce que l'exploitation des ressources naturelles du Sahara occidental (et pas seulement « énergétiques» comme dans la résolution du 27 octobre 2005) soit faite en conformité avec le droit international. Mais aussi, pour la première fois, le Parlement européen soutient l'élargissement du mandat de la MINURSO pour y inclure un élément de protection des droits de l'homme.

Finalement, durant l'année 2012, le Parlement européen a adopté deux résolutions concernant le Sahara occidental: la résolution du 18 avril $2012^{96}$ sur les droits de l'homme dans le monde et la politique de l'Union européenne en la matière, où l'on trouve un paragraphe $\left(n^{\circ}\right.$ 91) sur le Sahara occidental, qui demande la libération des personnes emprisonnées après la destruction du camp de protestation à Akdeim Izik et qui réitère la demande d'un mécanisme international pour la surveillance des droits de l'homme au Sahara occidental. Dans le même sens s'inscrit la résolution du 13 décembre $2012{ }^{97}$.

L'Assemblée paritaire de l'Union européenne et des pays ACP (Afrique, Caraïbes, Pacifique) s'est aussi quelques fois prononcée sur la question des droits de l'homme au Sahara

91. JOCE, C 33 E, 9 février 2006, p. 596-597.

92. JOCE, C 45 E, 23 février 2006, p. 107-129.

93. JOCE, C $272 \mathrm{E}, 9$ novembre 2006, p. 582-583.

94. Texte disponible à l'adresse: http://www.europarl.europa.eu/sides/getDoc.do?pubRef=-//EP//TEXT+MOTION+P7-RC-2009-0247+o+DOC+XML+Vo// FR

95. JOCE, C $99 \mathrm{E}, 3$ avril 2012, p. 87-88.

96. Résolution du Parlement européen du 18 avril 2012 sur le rapport annuel sur les droits de l'homme dans le monde en 2010 et la politique de l'Union européenne en la matière, notamment les implications pour la politique stratégique de l'Union européenne en matière de droits de l'homme, 2011/2185 (INI), disponible à l'adresse: http://www.europarl.europa.eu/sides/getDoc.do?type=TA\&reference=P7-TA-2012-0126\&language=FR.

97. Résolution du Parlement européen du 13 décembre 2012 concernant le rapport annuel 2011 sur les droits de l'homme et la démocratie dans le monde et la politique de l'Union européenne en la matière, 2012/2145 (INI), disponible à l'adresse: http://www.europarl.europa.eu/sides/getDoc. do type $=$ TA\&language $=F R \&$ reference $=\mathrm{P}_{7}-\mathrm{TA}-2012-503$. 
occidental. Cette Assemblée est le fruit de l'accord dit «Lomé III» et commença ses travaux durant l’année 1985.

Avec la résolution du 22 mars $1996^{98}$, adoptée à Windhoek, l'Assemblée paritaire ACP-UE - après avoir considéré les «accords tripartites de Madrid» comme une violation du droit international - «demande à l'UE de tenir compte des intérêts du peuple sahraoui dans les accords conclus avec le Royaume du Maroc». Cependant, la résolution de l'Assemblée paritaire ACP-UE du 26 septembre $1996^{99}$ ne parle plus des «intérêts » mais des «droits». Cette résolution porte attention aux droits politiques puisque «considérant les violations flagrantes des droits de l'homme perpétrés par les autorités marocaines au Sahara occidental», elle demande aux organes de l'UE et aux États membres d'user

[...] de tout leur pouvoir pour faire cesser les violations des droits de l'homme et obtenir la libération des centaines de Sahraouis toujours emprisonnés pour leurs convictions politiques.

Mais le plus important apport de cette résolution est sa référence aux droits économiques du peuple sahraoui. En effet, l'Assemblée « demande à l'Union européenne et aux États membres de tenir compte des droits inaliénables du peuple sahraoui dans les accords conclus avec le Maroc». Cependant, la résolution suivante de cette Assemblée sur le Sahara occidental, du 20 mars $1997^{100}$, même si elle persiste à considérer que les accords de Madrid violent le droit international, ne parle plus des droits de l'homme, qu'ils soient politiques ou économiques.

Les résolutions du Parlement européen condamnent d'une façon ferme les violations des droits de l'homme par le Maroc dans les territoires occupés du Sahara occidental. Mais l'Union européenne n'a pas une démarche cohérente vis-à-vis du sujet. Les incohérences de l'Union européenne sont particulièrement visibles dans les rapports entretenus avec le Maroc, notamment avec la signature de quelques traités qui ignorent les dénonciations faites par le Parlement européen, ou bien font de l'Union européenne la complice de certaines violations des droits du peuple sahraoui. C'est dans le domaine commercial, mais aussi dans le domaine politique, que l'on peut constater cette incohérence.
Entre 1988 et 2005, l'Europe et le Maroc ont signé quatre accords de pêche (1988, 1992, 1995 et 2005). Or, les eaux les plus poissonneuses qui constituent l'objet principal de l'accord (même s'il s'agit d'un non-dit) sont les eaux du Sahara occidental occupé.

En 1988, deux ans après l'adhésion de l'Espagne aux Communautés européennes, est signé le premier Accord sur les relations en matière de pêches maritimes entre la Communauté économique européenne et le Royaume du Maroc $^{101}$ pour une durée de quatre ans. Cet accord prévoit un maximum de huit cents licences annuelles pour les chalutiers européens (espagnols et portugais), sans aucune restriction sur les quantités pêchées ou les espèces. Cependant, le Maroc impose, en contrepartie, un délai d'un mois de repos biologique afin, dit-on, de "protéger» "ses» ressources halieutiques et obtient 282 millions d'écus. Un «repos biologique» qui existe seulement pour les chalutiers européens, mais non pour les chalutiers marocains, ni pour les chalutiers de certaines nationalités différentes de celles des pays de l'UE.

En 1992, est signé l'Accord sur les relations en matière de pêches maritimes entre la Communauté économique européenne et le Royaume du Maroc ${ }^{102}$, d'une durée de quatre ans, présentant de meilleures conditions pour le pays africain: la compensation financière est portée à 310 millions d'écus et la période de repos biologique prolongée à trois mois pour les céphalopodes et à deux mois pour les autres espèces. Les deux parties mettent fin à l'accord un an avant la date initialement fixée (avril 1995), en raison de désaccords sur l'utilisation des licences de pêche.

Cette même année, le Parlement rejette, dans une résolution commune du 15 janvier, les protocoles financiers avec la Syrie, le Maroc, l'Algérie, l'Égypte, la Tunisie, la Jordanie, le Liban et Israël parce que ces protocoles ne contiennent aucune disposition relative au respect des droits de l'homme ${ }^{103}$. La résolution indique qu'en «Syrie et au Maroc, notamment [...] les droits de l'homme sont violés d'une manière particulièrement grave». Cette résolution demande en outre à Israël de respecter les résolutions 242 et 338 du Conseil de sécurité et au Maroc d'appliquer la résolution 690 (celle qui approuvait le plan de règlement). La résolution souhaite qu'à l'avenir les

98. JOCE, C 254, 2 septembre 1996, p. 55

99. JOCE, C 62, 27 février 1997, p. 32-33.

100. JOCE, C 308,9 octobre 1997, p. 52.

101. Règlement (CEE) no 2054/88 du Conseil du 23 juin 1988 concernant la conclusion de l'accord sur les relations en matière de pêches maritimes entre la Communauté économique européenne et le Royaume du Maroc et arrêtant des dispositions pour son application (JOCE, L 181, 12 juillet 1988, p. 1-2); Accord sur les relations en matière de pêches maritimes entre la Communauté économique européenne et le Royaume du Maroc (JOCE, L 181, 12 juillet 1988, p. 3-13); Protocole $n^{\circ} 1$ fixant les possibilités de pêche accordées par le Maroc et la contrepartie accordée par la Communauté pour la période du $1^{\text {er }}$ mars 1988 au 29 février 1992 (JOCE, L 181, 12 juillet 1988, p. 14-16). Sur cet accord, voir J. Juste Ruiz, «El acuerdo pesquero CEE-Marruecos de 25 de febrero de 1988 ", Revista de instituciones europeas, n 15, septembre-décembre 1988, p. 741-764.

102. Règlement (CEE) no 3954/92 du Conseil du 19 décembre 1992 concernant la conclusion de l'accord sur les relations en matière de pêches maritimes entre la Communauté économique européenne et le Royaume du Maroc, et arrêtant des dispositions pour son application (JOCE, L 4O7, 31 décembre 1992, p. 1-2); Accord sur les relations en matière de pêches maritimes entre la Communauté économique européenne et le Royaume du Maroc (JOCE, L 407, 31 décembre 1992, p. 3-14); Protocole fixant les possibilités de pêche et les montants de la contrepartie financière et des appuis financiers accordés par la Communauté (JOCE, L 407, 31 décembre 1992, p. 15-28).

103. Résolution du 15 janvier 1992 sur les protocoles financiers avec la Syrie, le Maroc, l'Algérie, l'Égypte, la Tunisie, la Jordanie, le Liban et Israël et le respect des droits de l'homme et des accords internationaux par ces pays (JOCE, C 39, 17 février 1992, p. 50-52). 
protocoles d'accords prévoient une «clause démocratique et des droits de l'homme».

En 1995, est signé un Accord de coopération en matière de pêches maritimes entre la Communauté européenne et le Royaume du Maroc ${ }^{104}$, d'une durée de quatre ans, en conjonction avec l'accord d'association UE-Maroc. Le nouvel accord contient d'importantes modifications. Dès que, grâce aux importantes compensations financières octroyées par l'Union européenne, le Maroc aura développé sa propre industrie halieutique, est prévue une réduction progressive des licences délivrées par les autorités marocaines ainsi qu'une restriction quantitative de la pêche pour certaines espèces spécialement rentables (principalement des céphalopodes et des crevettes). En outre, la période de repos biologique pour les chalutiers européens est portée à quatre mois pour les céphalopodes et les autres types de poissons. La compensation financière s'élève à 355 millions d'écus à laquelle s'ajoute une aide de 145 millions d'écus pour le développement du secteur de la pêche industrielle, la recherche marine et la formation des pêcheurs marocains.

Après l'expiration en 1999 de l'accord de 1995, le Maroc fait savoir officiellement qu'il ne veut pas le renouveler. Cependant, la question est bien différente: primo, la prétendue volonté de non renouveler l'accord semble être un instrument de pression sur l'Espagne dans le conflit du Sahara occidental; et secundo, la valeur de l'accord pour le Maroc est devenue non seulement économique, mais plutôt politique, comme moyen de légitimation de l'occupation marocaine des eaux du Sahara occidental.
En 2006, malgré les prétendues difficultés de négociations ${ }^{105}$ pour un renouvellement de l'accord expiré en 1999, un nouvel accord de pêche, l'Accord de partenariat dans le secteur de la pêche entre la Communauté européenne et le Royaume du Maroc ${ }^{106}$ est signé. Certes moins important que les précédents accords, ce dernier est prévu, comme les autres, pour une durée de quatre ans. Il prévoit l'octroi de cent dix-neuf licences pour pêcher dans les eaux "marocaines» avec une contrepartie financière de 36 millions d'euros par an (soit 144 millions d'euros), outre les revenus issus de l'obligation de débarquer dans les ports «marocains» (y compris, donc, les ports du Sahara occidental) une partie des poissons capturés.

En 2011, après l'expiration de l'accord (entré en vigueur en février de 2007), le Conseil des ministres décide avec le Maroc une extension d'un an ${ }^{107}$. Mais en décembre 2011, le Parlement européen rejette l'extension entre autres raisons parce que l'accord inclut les eaux du Sahara occidental en violation du droit international ${ }^{108}$ et l'Union européenne décide d'abroger l'application provisoire de l'accord de pêche ${ }^{109}$.

L'Union européenne et le Maroc ont signé un accord d'association le 26 février 1996, entré en vigueur en $2000^{110}$. Le modèle de cet accord se fonde sur la prétention de ce qu'en échange de plusieurs avantages économiques de la partie européenne, la partie non européenne s'engage à améliorer sa situation politique en termes de démocratie, état de droit et respect des droits de l'homme. Comme il a été noté auparavant, il s'agissait d'une demande faite par le Parlement européen depuis 1992 au moins. Au vu des

104. Règlement (CE) n $\mathrm{n}^{\mathrm{0}}$ 150/97 du Conseil du 12 décembre 1996 concernant la conclusion de l'accord de coopération en matière de pêches maritimes entre la Communauté européenne et le Royaume du Maroc et arrêtant des dispositions pour son application (JOCE, L 30, 31 janvier 1997, p. 1-4); Accord de coopération en matière de pêches maritimes entre la Communauté européenne et le Royaume du Maroc (JOCE, L 30, 31 janvier 1997, p. 5-29). L'article 15 de l'accord dispose que le document est conclu "pour une durée de quatre ans à partir du $1^{\text {er }}$ décembre 1995 ".

105. Nous ne pouvons pas partager l'avis selon lequel le Maroc ne voulait pas reconduire l'accord de pêche de 1995 parce qu'il fut «a bad deal» pour le Maroc, comme l'affirme J. Vaquer i Fanés, «The Domestic Dimension of EU External Policies: The Case of the EU-Morocco $2000-01$ Fisheries Negotiations", Mediterranean Politics, vol. 8, n 1, 2003, p. 64. On doit noter que Vaquer parle toujours de "Moroccan waters» (passim) malgré le fait que les eaux les plus poissonneuses se trouvent au Sahara occidental occupé. En fait, l'auteur doit reconnaître qu'en application de l'accord de pêche le Maroc avait reçu « [an] enormous amount of money» (p. 69).

106. Règlement $(\mathrm{CE}) \mathrm{n}^{\circ} 764 / 2006$ du Conseil du 22 mai 2006 relatif à la conclusion de l'accord de partenariat dans le secteur de la pêche entre la Communauté européenne et le Royaume du Maroc (JOCE, L 141, 29 mai 2006, p. 1-3); Accord de partenariat dans le secteur de la pêche entre la Communauté européenne et le Royaume du Maroc (JOCE, L 141, 29 mai 2006, p. 4-37). Sur l'accord de pêche de 2006, voir E. Milano, «The New Fisheries Partnership Agreement between the European Community and the Kingdom of Morocco: Fishing Too South? ", Anuario de derecho internacional, vol. 22, 2006, p. 413-457; C. Ruiz-Miguel, «El acuerdo de pesca UE-Marruecos o el intento español de considerar a Marruecos como "potencia administradora" del Sahara Occidental», Anuario de derecho internacional, vol. 22, 2006, p. 395-412. Il est tout à fait étonnant de ne pas voir cités ces deux ouvrages dans le compte rendu de bibliographie sur les relations UE-Maroc de M. A. Sabiote, «Literature Review on the Relations between the EU and Morocco ", EUPROX State-of-the-arts Reports, n 3, juillet 2008. Peut-être pourrait-on expliquer cette omission par le fait que le choix de textes inclus est très favorable aux positions du régime marocain.

107. Décision 2011/491/UE du Conseil du 12 juillet 2011 relative à la signature, au nom de l'Union européenne, et à l'application provisoire du protocole entre l'Union européenne et le Royaume du Maroc fixant les possibilités de pêche et la contrepartie financière prévues par l'accord de partenariat dans le secteur de la pêche entre la Communauté européenne et le Royaume du Maroc (JOCE, L 202, 5 août 2011, p. 1-2); Protocole entre l’Union européenne et le Royaume du Maroc fixant les possibilités de pêche et la contrepartie financière prévues par l'accord de partenariat dans le secteur de la pêche entre la Communauté européenne et le Royaume du Maroc (JOCE, L 202, 5 août 2011, p. 3-30).

108. Texte disponible à l'adresse: http://www.europarl.europa.eu/news/fr/pressroom/content/20111213IPR34070/html/Accord-de-pêche-UE-Marocprorogation-rejetée-et-appel-à-un-meilleur-accord.

109. Décision 2012/15/UE du Conseil du 20 décembre 2011 abrogeant la décision 2011/491/UE du Conseil relative à la signature, au nom de l’Union européenne, et à l'application provisoire du protocole entre l'Union européenne et le Royaume du Maroc fixant les possibilités de pêche et la contrepartie financière prévues par l'accord de partenariat dans le secteur de la pêche entre la Communauté européenne et le Royaume du Maroc (JOCE, L 6, 10 janvier 2012, p. 1-2).

110. Décision 2000/204/EC, ECSC, du Conseil et de la Commission du 24 janvier 2000 relative à la conclusion de l'accord euro-méditerranéen établissant une association entre les Communautés européennes et leurs États membres, d'une part, et le Royaume du Maroc, d'autre part (JOCE, L 70, 18 mars 200o, p. 1; texte de l'accord p. 2-190). 
accords d'association signés par l'Union européenne et les événements subséquents, on peut conclure que le modèle n'a pas rencontré un grand succès ${ }^{111}$. Tous les accords d'association (y compris donc l'accord avec le Maroc) contiennent un article 2 dont la teneur est la suivante:

Le respect des principes démocratiques et des droits fondamentaux de l'homme, tels qu'énoncés dans la déclaration universelle des droits de l'homme, inspire les politiques internes et internationales de la Communauté et du Maroc et constitue un élément essentiel du présent accord.

Le problème qui se pose est celui d'une partie non européenne qui ne respecte pas cet engagement «essentiel». L'accord d'association prévoit l'établissement d'un Conseil d'association qui «examine les problèmes importants se posant dans le cadre de l'accord ainsi que toutes autres questions bilatérales ou internationales d'intérêt commun » (art. 78). D'après l'article 86 (1 et 2), « [c] haque partie peut saisir le Conseil d'association de tout différend relatif à l'application et à l'interprétation du présent accord» et "[1]e Conseil d'association peut régler le différend par voie de décision». Le problème est que ce Conseil d'association «arrête ses décisions et formule ses recommandations d'un commun accord entre les parties » (art. 8o). Bien sûr l'accord a également prévu la possibilité d'un différend entre les deux parties. Chaque partie peut alors notifier la désignation d'un arbitre à l'autre partie, qui est alors tenue de désigner un deuxième arbitre dans un délai de deux mois. Aux fins de l'application de cette procédure, la Communauté et les États membres sont considérés comme une seule partie au différend (art. 86.4). Mais, qui désigne le troisième arbitre? C'est le Conseil d'association qui doit le faire... et nous voilà encore face au problème de savoir comment on peut arriver à un accord lorsque l'on exige l'unanimité des deux parties. L'unique possibilité pour la partie qui estime qu'une éventuelle violation est tout à fait inacceptable consiste en la dénonciation du traité (art. 93).

En fait, malgré les résolutions du Parlement européen, rien ne s'est passé. Depuis l'entrée en vigueur de l'accord d'association, le Parlement européen a approuvé quelques résolutions qui dénoncent le fait que le Maroc a manqué à son engagement contenu dans l'article 2 de l'accord d'association et considéré comme un élément «essentiel». Il s'agit notamment des résolutions des 27 octobre $2005^{112}$ et 25 novembre $2010^{113}$. Malheureusement, rien ne s'est passé et le Conseil d'association ne s'est pas penché sur cet élément «essentiel».

Pis encore, trois semaines après la résolution du 25 novembre 2010, l'Union européenne et le Maroc ont signé, le 13 décembre 2010, un accord sur les mesures de libéralisation réciproques en matière de produits agricoles et de produits de la pêche qui modifie certains aspects de l'accord d'association de 1995 et qui octroie encore plus d'avantages commerciaux au Maroc. Cet accord est entré en vigueur en $2012^{114}$.

La question la plus étonnante tient au silence du Conseil de l'Europe face aux violations des droits de l'homme au Sahara occidental. L'étonnement est justifié par deux ordres de motifs : primo, parce que le but principal du Conseil de l'Europe est la défense et la promotion des droits de l'homme; et secundo, parce qu'un des États membres (le Royaume d'Espagne) est encore la puissance administrante de jure du territoire. Pour ces raisons, il semble bizarre que depuis que le Conseil d'Europe a décidé de se saisir de la question du Sahara occidental, il n'ait jamais exprimé sa préoccupation quant au problème du respect des droits de l'homme dans ce territoire. En effet, la résolution 1408 du 23 novembre $2004{ }^{115}$, adoptée par la Commission permanente de l'Assemblée consultative du Conseil de l'Europe, s'occupe du règlement politique de la question et on y trouve seulement un paragraphe traitant des personnes «toujours portées disparues dans le cadre du conflit» $(\$ 11)$.

\section{L'Union africaine et le respect des droits de l'homme au Sahara occidental: une réaction tardive, mais vigoureuse}

Le processus d'intégration régional en Afrique commence avec la création de l'Organisation de l'unité africaine (OUA) en 1963 et se poursuit avec la fondation de l'Union africaine (UA) en 2002.

La Charte de l'OUA est signée à Addis-Abeba le 25 mai 1963, aussi par le Maroc, qui était déjà un État indépendant. La Charte fait deux références aux droits de l'homme: le préambule indique que

[...] la Charte des Nations Unies et la Déclaration universelle des Droits de l'Homme, aux principes desquels nous réaffirmons notre adhésion, offrent une base solide pour une coopération pacifique et fructueuse entre nos États $[\ldots]$.

Et l'article II.1.e de la Charte déclare que parmi les objectifs de l'organisation figure la volonté de «favoriser la coopération internationale, en tenant dûment compte de la Charte des Nations Unies et de la Déclaration universelle des Droits de l'Homme».

111. L’Union européenne a des accords d'association avec la Tunisie (en vigueur en 1998), le Maroc et Israël (en vigueur en 20oo), la Jordanie (en vigueur en 2002), l'Égypte (en vigueur en 2004), l'Algérie (en vigueur en 2005) et le Liban (en vigueur en 2006).

112. JOCE, C 272 E, 9 novembre 2006, p. 582-583.

113. JOCE, C $99 \mathrm{E}, 3$ avril 2012, p. 87-88.

114. Décision 2012/497/UE du Conseil du 8 mars 2012 concernant la conclusion de l'accord sous forme d'échange de lettres entre l'Union européenne et le Royaume du Maroc relatif aux mesures de libéralisation réciproques en matière de produits agricoles, de produits agricoles transformés, de poissons et de produits de la pêche, au remplacement des protocoles $n^{\circ} 1,2$ et 3 et de leurs annexes et aux modifications de l'accord euroméditerranéen établissant une association entre les Communautés européennes et leurs États membres, d'une part, et le Royaume du Maroc, d'autre part (JOCE, L 241, 7 septembre 2012, p. 2 ; texte de l'accord p. 4-47).

115. Texte disponible à l'adresse: http://www.assembly.coe.int/ASP/Doc/XrefViewHTML.asp?FileID=17281\&Language=FR. 
L'Assemblée générale des Nations unies a demandé à l'Espagne depuis 1966 de «créer un climat politique favorable pour que le référendum se déroule sur des bases entièrement libres, démocratiques et impartiales ${ }^{116}$. L'OUA est allée encore un peu plus loin dans ses formules en demandant indirectement un respect des droits civils et politiques pour garantir le droit à l'autodétermination exigé depuis 1970 (avec le vote favorable du Maroc) ${ }^{117}$. En 1972, elle a demandé à l'Espagne de favoriser « une atmosphère libre et démocratique dans laquelle le peuple dudit territoire puisse exercer son droit d'autodétermination ${ }^{118}$. En 1973 et 1974, l'OUA a demandé à l'Espagne d'instaurer «un climat politique de liberté nécessaire à l'expression authentique de la volonté des populations ${ }^{119}$.

Le 27 février 1976, au lendemain de la retraite de l'Espagne du territoire, se proclame la République arabe sahraouie démocratique (RASD). Elle est admise au sein de l'OUA en 1982, mais sa participation effective est provisoirement retardée, en attendant l'accomplissement par le Maroc de sa promesse, solennellement formulée à l'OUA en 1981, d'organiser le référendum d'autodétermination du peuple du Sahara occidental. Mais le Maroc ne met pas en place le référendum et la République sahraouie prend effectivement son siège à l'OUA en 1984. Le Maroc annonce ensuite son retrait de l'OUA.

En 1981, les États membres de l'OUA signent à Nairobi la Charte africaine des droits de l'homme et des peuples qui entre en vigueur le 21 octobre 1986 après sa ratification par vingt-cinq États parmi lesquels se trouve la République sahraouie. Depuis 1988, la République sahraouie a présenté les rapports exigés par l'article 62 de la Charte des droits de l'homme. Cependant, la Commission africaine des droits de l'homme et des peuples (CADHP) chargée de veiller au respect de la Charte ne s'est prononcée sur la situation au Sahara occidental qu'en 2000, en faisant seulement référence à la nécessité de respecter le droit à l'autodétermination du peuple sahraoui ${ }^{120}$.

Le 11 juillet 2000, les cinquante-trois membres de l'OUA décident de dépasser le modèle d'intégration régionale mis en place en 1963 pour initier un nouveau processus d'intégration inspiré par le modèle européen, comme le démontre le nom même de l'organisation, l'Union africaine (UA). L'Acte constitutif de l'UA entre en vigueur le 9 juillet 2002. Il porte un peu plus d'attention aux droits de l'homme (préambule, articles 3.e, 3.h et 4.m) que la Charte de l'OUA.

C'est seulement plusieurs années après l'entrée en vigueur de l'Acte constitutif de l'UA que l'UA traite la question des droits de l'homme au Sahara occidental, au-delà du droit à l'autodétermination. En janvier 2012, le Conseil exécutif de l'UA a approuvé la décision de dépêcher une mission de la CADHP au Sahara occidental, y compris dans les territoires occupés par le Maroc pour enquêter sur la situation des droits de l'homme ${ }^{121}$. La CADHP avait annoncé que sa mission devait se dérouler du 24 au 28 septembre $2012^{122}$, mais elle n'a pu se rendre que dans une partie du territoire libéré et dans les camps de réfugiés, et non dans les territoires occupés. Le Royaume du Maroc n'a en effet pas répondu à la demande de la Commission relative au passage en territoires occupés ${ }^{123}$.

La CADHP a cependant réagi très vite au jugement de plusieurs citoyens sahraouis détenus après la destruction par le Maroc du camp de protestation érigé à Akdeim Izik, près du Maroc en octobre-novembre $2010^{124}$.

\section{VII.Les Nations unies: une réaction tardive et difficile face aux violations des droits de l'homme au Sahara occidental}

La responsabilité des Nations unies envers le peuple du Sahara occidental a été implicitement établie en 1967 et expressément en $1972^{125}$. Elle a été réitérée en 1975 après la signature des soi-disant "accords de Madrid» qui avaient établi une soi-disant «administration intérimaire tripartite ${ }^{126}$ et a été constamment proclamée jusqu’à nos jours ${ }^{127}$.

Les Nations unies se sont tardivement penchées sur la question des droits de l'homme au Sahara occidental.

116. Résolution de l'Assemblée générale A/RES/2229 (XXI) du 20 décembre 1966 (nous soulignons).

117. Voir S. Omar, «OAU / AU and the Question of Western Sahara», Pambazuka News, n 618, 27 février 2013, disponible à l'adresse: http:// pambazuka.org/en/category/features/86402.

118. Conseil des ministres de l'OUA, résolution CM/Res. 272 (XIX) du 5 au 12 juin 1972.

119. Conseil des ministres de l'OUA, résolutions sur le Sahara sous domination espagnole, documents CM/Res. 344 (XXI) du 17 au 24 mai 1973 et CM/Res. 344 (XXIII) du 6 au 11 juin 1974.

120. Résolution 45 sur le Sahara occidental de la Commission africaine des droits de l'homme et des peuples, réunie en sa $27^{\mathrm{e}}$ session ordinaire à Alger, du 27 avril au 11 mai 2000, disponible à l'adresse: http://www.achpr.org/fr/sessions/27th/resolutions/45.

121. EX.CL/Dec.689(XX), disponible à l'adresse: http://www.peaceau.org/uploads/ex-cl-dec-689-XX-f.pdf: «Le Conseil exécutif: [...] 10. demande à la CADHP d'effectuer une mission dans les territoires occupés de la République arabe sahraouie démocratique (RASD) en vue d'enquêter sur les violations des droits de l'homme et de faire rapport à la prochaine session ordinaire du Conseil exécutif en janvier 2013".

122. Communiqué de presse sur la mission d'établissement des faits en République arabe sahraouie démocratique, 17 septembre 2012 , disponible à l'adresse: http://www.achpr.org/fr/press/2012/o9/d13o.

123. Communiqué de presse sur la mission d'établissement des faits de la Commission africaine des droits de l'homme et des peuples en République arabe sahraouie démocratique, 28 septembre 2012, disponible à l'adresse: http://www.achpr.org/fr/press/2012/10/d135.

124. Déclaration de la Commission africaine des droits de l'homme et des peuples sur le procès et la condamnation de vingt-cinq civils sahraouis par un tribunal militaire marocain, 23 février 2013, disponible à l'adresse: http://www.achpr.org/fr/press/2013/02/d142.

125. A/RES/2229 du 20 décembre 1966; A/RES/2983 du 14 décembre 1972 et A/RES/3162 du 14 décembre 1973.

126. A/RES/3458-A du 10 décembre 1975.

127. A/RES/64/101 (2010), A/RES/65/112 (2011), A/RES/66/86 (2012), A/RES/67/129 (2013). 
L'attention des différents organes du système des Nations unies semble grandissante ces dernières années, mais elle se heurte à une grande difficulté pour enquêter et à une impossibilité d'empêcher les violations des droits de l'homme.

Lorsque l'on étudie le travail accompli par le système des Nations unies sur la situation des droits de l'homme au Sahara occidental, il faut examiner différents points: le travail du secrétaire général, de l'Assemblée générale et du Conseil de sécurité (A); ce qu'ont ensuite réalisé la Commission des droits de l'homme, le Comité des droits de l'homme, le Conseil des droits de l'homme et le Comité contre la torture (B) ; et finalement la possibilité d'une éventuelle intervention de la Cour pénale internationale (C).

\section{A. L'Assemblée générale, le secrétaire général et le Conseil de sécurité}

Les rapports du secrétaire général et les résolutions de l'Assemblée générale et du Conseil de sécurité des Nations unies sur le Sahara occidental ont été longtemps centrés sur la question de la décolonisation et la mise en œuvre d'une solution pour permettre l'autodétermination du peuple sahraoui.

L'Assemblée générale ne s'est penchée qu'une seule fois sur une question connectée aux droits de l'homme. Il s'agit de sa résolution de 1970, qui « regrette les incidents sanglants survenus dans le territoire en juin 1970» lors de la répression d'une manifestation ${ }^{128}$. Il est étonnant de constater qu'aucune autre résolution de l'Assemblée générale ne s'est penchée sur des questions relatives au respect des droits de l'homme bien que des incidents et situations encore plus graves que ceux de 1970 se soient produits dans le territoire.

On pourrait dire que la première fois où la question des droits de l'homme a été évoquée dans un rapport du secrétaire général, même de manière implicite, c'est lorsque le secrétaire général a présenté les propositions du plan de règlement agréé par les deux parties au conflit, le Royaume du Maroc et le Front Polisario. Le plan, approuvé par le Conseil de sécurité, prévoit quelques dispositions qui concernent les droits de l'homme dans la dénommée "période transitoire" s'étendant de l'instauration du cessez-le-feu à la proclamation des résultats du référendum d'autodétermination. Pendant cette période, le représentant spécial des Nations unies est la seule et exclusive autorité pour toutes les questions relatives au référendum, son organisation et son contrôle ${ }^{129}$. Le plan de règlement dispose que:
Afin de remplir pleinement et efficacement le mandat et les fonctions qui lui sont confiés, le Représentant spécial sera habilité à prendre toutes les mesures qu'il jugera nécessaires pour garantir la liberté de mouvement et la sécurité de la population, ainsi que l'impartialité du référendum. À cette fin, il sera autorisé à prendre sur place toutes les mesures d'ordre administratif, technique ou de sécurité qu'il jugera bon d'appliquer dans le territoire pendant la période transitoire. Ces mesures porteront sur des questions relatives au déroulement de la campagne référendaire telles que les réunions politiques et la publicité. Le Représentant spécial du Secrétaire général pourra également demander la suspension de toute loi ou mesure qui, à son avis, pourrait entraver le bon déroulement du référendum libre et équitable. Tout différend qui interviendrait entre les parties au conflit et le Représentant spécial devra être soumis à la décision du Secrétaire général de l'ONU ${ }^{130}$.

Bien que la période transitoire ait commencé avec le cessez-le-feu du 6 septembre 1991, la disposition mentionnée du plan de règlement n'a jamais été invoquée par le représentant spécial des Nations unies.

La question des droits de l'homme a acquis davantage d'ampleur dans les rapports du secrétaire général lorsque le processus de la décolonisation s'est heurté à des blocages. La première référence directe aux droits de l'homme apparaît en 2001, après que le Maroc a bloqué l'application du référendum qui devrait être organisé d'après le plan de règlement (dont l'une des prévisions était la libération des prisonniers de guerre). En avril 2001, le secrétaire général a exprimé sa préoccupation sur le sort des prisonniers de guerre, considéré comme «une question de droits de l'homme " ${ }^{131}$. Quelques mois après, le secrétaire général, inspiré par le Maroc, a présenté une proposition qui, tout en annexant le Sahara occidental au Maroc, prévoit une autonomie garantie internationalement ${ }^{132}$. Le paragraphe 4 de cette proposition précise que

[t] outes les lois promulguées par l'Assemblée et toutes les décisions des tribunaux visées au paragraphe 3 ci-dessus doivent être conformes à la Constitution du Royaume du Maroc et en respecter les dispositions, en particulier en ce qui concerne la protection des libertés publiques.

Au même moment, face à la préoccupation liée aux problèmes humanitaires du conflit manifestée par le président du Conseil de sécurité le 26 avril 2001, le Front Polisario proposa «le respect des droits de l'homme sur le Territoire et son ouverture aux organismes humanitaires et aux médias étrangers ${ }^{133}$.

Cependant, c'est en 2003 que la question des droits de l'homme reçut pour la première fois une attention spécifique et détaillée du secrétaire général et fit l'objet d'un

128. A/RES/2711 (XXV) du 14 décembre 1970.

129. «Propositions présentées par le Secrétaire général de l’Organisation des Nations unies et le Président en exercice de la Conférence des chefs d’État et de gouvernement de l'Organisation de l'unité africaine en vue de régler la question du Sahara occidental et acceptées en principe par les parties le 30 août 1988 », première partie du rapport du secrétaire général au Conseil de sécurité du 18 juin $1990(\mathrm{~S} / 21360)$, $\$ 8$ et 50 .

130. Ibid., $\$ 10$.

131. S/2001/398 du 24 avril, $\$ 9$.

132. "Accord-cadre sur le statut du Sahara occidental», annexe I du rapport du secrétaire général, S/2001/613 du 20 juin 2001.

133. Annexe IV du rapport du secrétaire général, $S / 2001 / 613$ du 20 juin 2001. 
débat. Le plan Baker présenté par le secrétaire général ${ }^{134}$ contient deux dispositions sur la question des droits de l'homme:

13. Les lois, règlements et autres textes adoptés par l'Autorité du Sahara occidental doivent tous être conformes aux normes internationales relatives aux droits de l'homme (y compris les normes relatives aux droits de l'homme prévues par tout traité auquel le Maroc est partie). En aucun cas la protection des droits de l'homme au Sahara occidental ne doit être moindre que celle qui est prévue par la Constitution et les lois du Maroc.

[...]

17. Les campagnes électorale et référendaire prévues dans le présent plan seront menées dans le respect des normes internationales relatives aux droits de l'homme.

Ces dispositions n'étaient complètement acceptables ni pour le Front Polisario ni pour le Maroc, pour des raisons opposées. Le Front Polisario considérait qu'il était également nécessaire de renforcer l'internationalisation du régime d'autonomie. Il déclara que

[e]n prévoyant de concéder au Maroc (dont les pratiques policières sont bien connues) l'administration des postes et télécommunications, la proposition met en péril le respect du principe universel de la liberté et du secret des communications et des correspondances. Cela reviendrait à une remise en cause des droits fondamentaux de l'homme au détriment des Sahraouis ${ }^{135}$.

Le Maroc, quant à lui, manifesta son opposition au plan Baker, et particulièrement aux dispositions sur les droits de l'homme, pour des raisons tout à fait contraires: selon lui les garanties des droits de l'homme devraient reposer sur le mécanisme institutionnel marocain. Primo, le Royaume a contesté l'établissement d'un régime d'autonomie (transitoire dans le plan Baker) surveillé internationalement parce que

[...] la mise en place d'un statut d'autonomie dans le cadre de la structure étatique existante [...] est [...] le meilleur garant du respect des droits fondamentaux de la personne humaine reconnus par la Constitution marocaine et confortés par les engagements internationaux du Royaume ${ }^{136}$.

Secundo, suivant cette ligne argumentaire, le Royaume du Maroc a précisé que «la justice est rendue au nom de Sa Majesté le Roi, protecteur des droits et libertés des citoyens en vertu de l'article 19 de la Constitution». Et tertio que l'«on aurait souhaité [...] que le système judiciaire du Royaume soit pleinement habilité à veiller au respect de ces droits $»^{137}$
Cependant, les arguments du Royaume du Maroc sont peu soutenables. En fait, en 2004, l'année suivant la présentation de ces allégations, l'IER, organisme officiel marocain a reconnu que ni le roi («protecteur des droits et libertés des citoyens en vertu de l'article 19 de la Constitution »), ni le système judiciaire du Royaume n'avaient veillé à protéger les droits de l'homme jusqu'en 1999. En plus, rien ne laissait penser que le roi ou le système judiciaire du Royaume allaient changer d'attitude. Comme précédemment observé, parmi toutes les violations des droits de l'homme au Sahara occidental dénoncées depuis 1975, il n'existe qu'un cas (celui de Hamdi Lembarki) qui a reçu une sanction officielle, mais très légère.

Le changement de gouvernement en Espagne après les attentats du 11 mars 2004 a été suivi d'un revirement radical dans la politique sur le Sahara occidental, et a conduit James Baker à démissionner en juin 2004. Le nouveau blocage du processus de règlement du conflit explique le déclenchement, le 21 mai 2005, de la dénommée «intifada de l'indépendance" suivie d'une vague de répression par le gouvernement marocain. Pour contrecarrer les dénonciations de violations des droits de l'homme commises par le royaume marocain, celui-ci à son tour a déclenché une campagne pour dénoncer des prétendues violations des droits de l'homme par le Front Polisario dans les camps de réfugiés de Tindouf. Le secrétaire général de l'ONU, rappelant que la MINURSO ne bénéficiait pas de compétences dans le domaine des droits de l'homme, a exprimé sa préoccupation au sujet des allégations faisant état de violations des droits de l'homme par les parties ${ }^{138}$. La persistance des protestations sahraouies et de la répression a poussé le secrétaire général du Front Polisario à demander l'intervention des Nations unies pour la protection des droits de l'homme au Sahara occidental. Au vu des dénonciations croisées des deux parties, le secrétaire général des Nations unies a proposé l'envoi d'une mission du Haut-Commissariat des Nations unies aux droits de l'homme (HCNUDH) pour s'enquérir de la situation et proposer des mesures à prendre par les Nations unies pour mieux répondre aux préoccupations exprimées en la matière ${ }^{139}$. La mission du HCNUDH s'est déroulée du 15 au 23 mai, et le 19 juin 2006. Concernant la situation des droits de l'homme, le rapport du HCNUDH confirme les dénonciations faites par le Front Polisario et constate que les autorités marocaines sont responsables des violations des droits de l'homme ${ }^{140}$. Cependant, le HCNUDH ne confirme pas les dénonciations du Royaume du Maroc contre le

134. «Plan de paix pour l'autodétermination du peuple du Sahara occidental», annexe II du rapport du secrétaire général, S/2003/565 du 23 mai 2003.

135. Lettre datée du 8 mars 2003, adressée au secrétaire général de l'ONU par le secrétaire général du Front Polisario, annexe III du rapport du secrétaire général $\mathrm{S} / 2003 / 565$ du 23 mai 2003.

136. "Observations du Royaume du Maroc sur la nouvelle proposition de James Baker intitulée: "Plan de paix pour l'autodétermination de la population du Sahara occidental" ", annexe III du rapport du secrétaire général, S/2003/565 du 23 mai 2003.

137. Ibid.

138. S/2005/648 du 13 octobre 2005 .

139. $S_{72006 / 249}$ du 19 avril, $\$ 3$ et 41 .

140. Haut-Commissariat des Nations unies aux droits de l'homme, Report of the OHCHR Mission to the Western Sahara and the Refugee Camps in Tindouf. 15/23 May and 19 June 2006, Genève, HCNUDH, 8 septembre 2006, \$11-36. Le rapport n'a pas été publié mais a été diffusé. 
Front Polisario ${ }^{141}$. Concernant les mesures proposées par le HCNUDH pour améliorer la situation des droits de l'homme, le Haut-Commissariat précise que «vu la gravité de la situation des droits de l'homme au Sahara occidental», une vigilance plus étroite et continue s'avère «indispensable», s'accompagnant d'une capacité effective pour protéger contre les violations et corriger celles-ci ${ }^{142}$.

Depuis octobre $2006^{143}$, la question des droits de l'homme est devenue (sans interruption jusqu'à aujourd'hui) l'un des chapitres clefs des rapports du secrétaire général des Nations unies sur le Sahara occidental. En avril 2011, il a considéré l'idée d'exploiter plus pleinement les possibilités offertes par les mécanismes du Conseil des droits de l'homme. Il attend également que les mécanismes du Haut-Commissariat pour les réfugiés s'engagent de leur côté à examiner de façon «indépendante, impartiale et soutenue» les allégations faisant état de violations des droits universels du peuple du Sahara occidental dans le territoire et dans les camps ${ }^{144}$. Dans son rapport d'avril 2012, le secrétaire général, tout en réitérant son appel à faire usage des possibilités offertes par les mécanismes du Conseil des droits de l'homme, a indiqué qu'il était «trop tôt » pour mesurer pleinement l'impact des mécanismes de contrôle du Conseil des droits de l'homme dans ce contexte, mais que certaines limites étaient déjà apparentes, en raison de la portée du rôle des titulaires de mandats relevant des procédures spéciales et de la structure de l'action qu'ils mènent ${ }^{145}$.

Cependant, en 2013, le secrétaire général a avoué l'insuffisance des mécanismes du Conseil des droits de l'homme pour traiter la question du Sahara occidental. Dans son rapport, il a à nouveau pressé pour l'établissement d'un mécanisme de surveillance des droits de l'homme spécifique pour le Sahara occidental:

Comme les violations des droits de l'homme n'ont apparemment pas cessé, la surveillance indépendante, impartiale, complète et constante de la situation des droits de l'homme au Sahara occidental et dans les camps devient plus que jamais une nécessité primordiale ${ }^{146}$.

Le Front Polisario a demandé depuis 2006 que la MINURSO soit dotée d'une composante droits de l'homme ${ }^{147}$ tout comme d'autres missions des Nations unies ${ }^{148}$. Le Maroc a rejeté cette possibilité et le Conseil de sécurité a évité de se prononcer sur la question.

Le Conseil de sécurité donnant son approbation au plan de règlement ${ }^{149}$ avait investi le représentant spécial des Nations unies pour le Sahara occidental de pouvoirs dans le domaine des droits de l'homme, non explicitement mentionnés, mais clairement évoqués («le Représentant spécial sera habilité à prendre toutes les mesures qu'il jugera nécessaires pour garantir la liberté de mouvement et la sécurité de la population ${ }^{150}$ ).

Cependant, après l'approbation du plan de règlement les références explicites sur la question des droits de l'homme ne sont apparues qu'au bout de presque vingt ans. Il a fallu attendre l'année 2009 pour relever une vague référence à l'importance d'effectuer des progrès dans la «dimension humaine» du conflit ${ }^{151}$. La référence à la dimension humaine a été introduite par l'Autriche, alors membre non permanent du Conseil de sécurité comme une référence voilée aux droits de l'homme, car dans le cadre de l'Organisation pour la sécurité et la coopération en Europe (OSCE), dont le siège est à Vienne, l'expression «dimension humaine» est employée pour faire référence aux droits de l'homme. À ce moment (2009) existait un intense débat sur la question des droits de l'homme au Sahara occidental, mais le Royaume du Maroc et ses alliés au Conseil de sécurité ont refusé qu'une quelconque référence à ce sujet soit faite dans la résolution du Conseil. L'expression « dimension humaine» était alors une solution de compromis.

Cependant, la situation des droits de l'homme au Sahara occidental s'est dégradée les mois et années suivants. En novembre 2009, le Maroc a décidé la déportation de l'ancienne prisonnière politique et défenseure des droits de l'homme, Aminatou Haïdar, mais après une retentissante grève de la faim de $\mathrm{M}^{\text {me }}$ Haïdar et des pressions étrangères (provenant notamment des États-Unis) le Royaume a annulé la déportation. L'année suivante, en octobre 2010, les Sahraouis ont organisé un camp de protestation massif à Akdeim Izik près de la capitale du Sahara occidental (El-Aaiun), violemment détruit le 8 novembre 2010. Après ces événements, en 2011, le Conseil de sécurité

141. Ibid., $\$ 37-51$.

142. Ibid., $\$ 57$.

143. $\mathrm{S} / 2006 / 817$ du 16 octobre 2006 .

144. $\mathrm{S} / 2011 / 249 \mathrm{du} 1^{\text {er }}$ avril $2011, \$ 121$.

145. S/2012/197 du 5 avril 2012, $\$ 106$ et 84. En avril 2012, on comptait trente-six titulaires de mandats thématiques portant sur une vaste gamme de questions relatives aux droits de l'homme, chacun ayant la mission de rendre compte de la situation concernant les droits de l'homme, de fournir des conseils à ce sujet dans une perspective thématique et d'examiner la situation dans l'ensemble des États membres de l'ONU. Chaque rapporteur ne peut toutefois se rendre que dans deux ou trois États par an.

146. $S / 2013 / 220$ du 9 avril 2013, $\$ 116$ (nous soulignons).

147. Document disponible à l'adresse: http://www.wshrw.org/supervision-de-los-derechos-humanos-en-el-sahara-occidental-posiciones-del-frentepolisario.

148. Afghanistan (S/RES/1401), Haïti (S/RES/1542), Soudan (S/RES/1590), Sierra Leone (S/RES/1620), Népal (S/RES/1740).

149. S/RES/658 du 27 juin 1990; S/RES/69o du 29 avril 1991.

150. «Propositions présentées par le Secrétaire général de l'Organisation des Nations unies et le Président en exercice de la Conférence des chefs d'État et de gouvernement de l'Organisation de l'unité africaine en vue de régler la question du Sahara occidental et acceptées en principe par les parties le 30 août 1988 ", première partie du rapport du secrétaire général au Conseil de sécurité du 18 juin 1990 (S/21360), approuvé par la résolution 658 du Conseil de sécurité, du 27 juin 1990, $\$ 10$.

151. S/RES/1871 du 30 avril 2009 et, dans le même sens, S/RES/1920 du 30 avril 2010. 
a effectué un pas en avant et introduit, pour la première fois, une référence aux « droits de l'homme». La résolution souligne "qu'il importe d'améliorer la situation des droits de l'homme au Sahara occidental et dans les camps de Tindouf» et engage les parties

[...] à collaborer avec la communauté internationale pour mettre au point et appliquer des mesures indépendantes et crédibles qui garantissent le plein respect des droits de l'homme, en gardant à l'esprit les obligations découlant du droit international ${ }^{152}[\ldots]$.

Mais elle n'établit pas de dispositif international spécifique pour veiller à cette amélioration. Elle salue toutefois l'engagement pris par le Maroc d'

[...] accorder un accès sans réserves ni restrictions à tous les titulaires de mandats relevant des procédures spéciales $\mathrm{du}$ Conseil des droits de l'homme ${ }^{153}[\ldots]$.

En 2013, un pas en avant qualitatif a semblé survenir avec, suite à la pétition formulée par le secrétaire général (voir supra), la mise en place d'un mécanisme «indépendant, impartial, complet et constant» pour la surveillance des droits de l'homme au Sahara occidental et dans les camps de réfugiés. La presse électronique a fait état de la présentation par les États-Unis d'un brouillon de résolution prévoyant l'attribution à la MINURSO des compétences pour surveiller et rapporter les violations des droits de l'homme au Sahara occidental et dans les camps de Tindouf. Cependant, plusieurs États (notamment la France et l'Espagne) appartenant au dénommé Groupe des amis du Sahara occidental (composé des États-Unis, du Royaume-Uni, de la France, de la Russie et de l'Espagne comme puissance administrante de jure du territoire) s'y sont opposés. Finalement, les États-Unis ont retiré leur premier brouillon et la résolution adoptée réitère avec de petits changements terminologiques les formules assumées depuis 2011 et ajoute que le Conseil engage les parties (Royaume du Maroc et Front Polisario)

[...] à poursuivre les efforts qu'elles mènent chacune pour renforcer la promotion et la protection des droits de l'homme au Sahara occidental et dans les camps de réfugiés de Tindouf ${ }^{154}$.

\section{B. Les organes des Nations unies spécialisés dans le domaine des droits de l'homme}

La Commission des droits de l'homme, le Comité des droits de l'homme, le Comité des droits économiques, sociaux et culturels, et le Conseil des droits de l'homme ont joué un rôle très limité dans le domaine des droits de l'homme au Sahara occidental, tout comme le Comité contre la torture.

La Commission des droits de l'homme a été établie par la résolution 5 (I) du Conseil économique et social du 16 février 1946. La Commission se réunissait en session annuelle et, si nécessaire, en session spéciale et rapportait au Conseil économique et social. De 1994 à 2004, la Commission des droits de l'homme a émis des résolutions sur le Sahara occidental mais ces résolutions n'ont traité que du droit à l'autodétermination ${ }^{155}$.

Le Comité des droits de l'homme a eu l'occasion d'adresser quelques remarques critiques au Royaume du Maroc concernant la situation des droits de l'homme au Sahara occidental.

En 1991, dans son premier rapport sur le Maroc, le Comité a posé plusieurs questions sur les droits de l'homme au Sahara occidental.

La première concerne les disparus. Le Comité a demandé

[...] si des prisonniers politiques originaires du Sahara occidental avaient disparu ou étaient morts en prison et, dans l'affirmative, si des enquêtes avaient été réalisées dans de tels cas ${ }^{156}$

En deuxième lieu, «quelle était la justification des mesures de contrôle strictes appliquées aux étrangers, en particulier aux étrangers de langue espagnole au Sahara occidental ${ }^{157}$ concernant la liberté de circulation et expulsion des étrangers.

En troisième lieu, des membres du Comité ont

[...] exprimé à nouveau leurs inquiétudes au sujet des arrestations arbitraires, des disparitions, des conditions de détention, de l'existence de prisons non reconnues, de la longueur parfois excessive de la durée de détention, des problèmes relatifs à l'indépendance du système judiciaire et à l'application de certains aspects de l'article 14 du Pacte, notamment la préparation d'une défense et la charge de la preuve, de la suppression des activités politiques et culturelles dans les universités ainsi que d'autres restrictions dans le domaine de la liberté d'expression, comme le fait de ne pas autoriser la critique des institutions marocaines ou de la monarchie, de la façon dont sont traités les habitants du Sahara occidental et de la situation difficile de la communauté bahaie ainsi que des restrictions à la liberté de religion en général ${ }^{158}$.

Le gouvernement marocain a répondu à la première question que «les autorités avaient été informées d'un certain nombre de demandes de dossiers concernant des personnes disparues au Sahara occidental et qu'elles examinaient tous ces cas avec le plus grand soin ${ }^{159}$. Il a

152. S/RES/1979 du 27 avril 2011, \$11 du préambule.

153. Ibid., $\$ 12$ du préambule; dans le même sens, voir S/RES/2044 du 24 avril 2012 qui reproduit ces clauses.

154. S/RES/2099 du 25 avril 2013, \$14 du préambule.

155. Résolutions de la Commission des droits de l'homme 1994/6, 1995/7, 1996/6, 1997/5, 1998/5, 1999/4, 2000/2, 2001/1, 2002/4, 2003/1 et 2004/4.

156. Rapport du Comité des droits de l'homme, A/47/40(SUPP), 9 octobre 1992, $\$ 52$.

157. Ibid., $\$ 63$.

158. Ibid., $\$ 77$.

159. Ibid., $\$ 56$. 
également ajouté que «plusieurs disparitions remontaient à une époque où le Maroc n'administrait pas le Sahara occidental» et qu'il y avait aussi «des problèmes d'identification, de noms et d'orthographe, étant donné que les nomades en question n'étaient souvent identifiés que par leur appartenance tribale». Cette réponse du gouvernement a été décrédibilisée par les autorités marocaines elles-mêmes après la publication du rapport de l'IER en 2005.

Dans ses observations au rapport présenté par le Maroc en 1994, le Comité a exprimé sa préoccupation concernant le rôle du Maroc dans les problèmes posés au droit à l'autodétermination du peuple sahraoui (article 1 du Pacte international relatif aux droits civils et politiques - PIDCP) et vis-à-vis des mesures de grâce adoptées par le roi dans la période examinée qui n'ont généralement pas bénéficié aux Sahraouis ${ }^{160}$.

Dans ses observations au rapport présenté par le Maroc en 1999, le Comité est demeuré préoccupé par la lente préparation du référendum d'autodétermination au Sahara occidental et l'absence d'information sur la mise en pratique des droits de l'homme dans ce territoire. Il a demandé au Maroc de coopérer pour compléter les opérations conduisant au référendum ${ }^{161}$.

Dans ses observations au rapport présenté par le Maroc en 2004, le Comité était à nouveau préoccupé par l'absence de progrès dans l'organisation du référendum d'autodétermination du peuple sahraoui et par le fait que plusieurs membres d'organisations non gouvernementales sahraouies avaient été empêchés de se rendre à Genève pour assister à la $49^{\mathrm{e}}$ session de la Commission des droits de l'homme (en violation des articles 12 et 19 du PIDCP) ${ }^{162}$.

Le Comité des droits économiques, sociaux et culturels a également formulé des observations sur le respect des droits au Sahara occidental.

En 1994, dans l'examen du rapport initial présenté par le Maroc suite à la ratification du Pacte international des droits économiques, sociaux et culturels (PIDESC), le Comité a examiné le respect du droit à l'autodétermination non seulement sous son aspect politique, mais aussi sous son aspect économique, social et culturel et noté avec préoccupation qu'au Sahara occidental

[...] le droit à l'autodétermination n'a pas été exercé en parfaite conformité avec les dispositions de l'article $1 \mathrm{du}$ Pacte et selon les plans approuvés par le Conseil de sécurité de l'Organisation des Nations Unies

et s'est déclaré
[...] préoccupé par les conséquences négatives de la politique du Maroc au Sahara occidental sur la jouissance des droits économiques, sociaux et culturels de la population concernée, en particulier du fait d'un transfert de populations ${ }^{163}$.

Dans les observations publiées en 2000, le Comité s'est seulement penché sur l'aspect politique du droit à l'autodétermination et a « regrett[é] qu'une solution claire n'ait pas été apportée à la question de l'autodétermination $~^{164} \mathrm{en}$

[...] encourage[ant] l'État partie à trouver une solution, en coopération avec l'Organisation des Nations Unies, aux problèmes qui entravent le processus de référendum sur la question de l'autodétermination du Sahara occidental ${ }^{165}$.

En 2006, le Comité, tout en réitérant sa préoccupation sur la question de l'autodétermination, a pris note

[...] avec inquiétude d'informations faisant état de la situation précaire des personnes déplacées suite au conflit du Sahara occidental, en particulier les femmes et les enfants, qui seraient victimes de violations multiples des droits qui leur sont reconnus dans le Pacte $^{166}[\ldots]$.

Et a «pri[é] l'État partie de prendre des mesures pour protéger les droits des personnes déplacées suite au conflit du Sahara occidental, et de leur apporter la sécurité ${ }^{167}$.

Le Conseil des droits de l'homme a été créé par l'Assemblée générale de l'ONU en $2006^{168}$, avec le but principal d'aborder des situations de violations des droits de l'homme et d'émettre des recommandations à leur encontre. Ce Conseil se substitue à la Commission des droits de l'homme et exerce ses fonctions de deux manières: une procédure "ordinaire » qui consiste en un "examen périodique universel» (universal periodic review - UPR) des pays membres des Nations unies; des "procédures spéciales " héritées de la pratique de la Commission des droits de l'homme, notamment la nomination de certains «rapporteurs spéciaux».

La compétence principale et ordinaire du Conseil est de procéder à un examen périodique universel,

[...] sur la foi d'informations objectives et fiables, du respect par chaque État de ses obligations et engagements en matière de droits de l'homme, de façon à garantir l'universalité de son action et l'égalité de traitement de tous les États; se voulant une entreprise de coopération fondée sur un dialogue auquel le pays concerné est pleinement associé et qui tient compte des besoins de ce dernier en termes de renforcement de ses capacités, cet examen viendra compléter l'œuvre des organes conventionnels sans faire double emploi ${ }^{169}[\ldots]$.

160. Observations du Comité des droits de l'homme: Maroc, CCPR/C/79/Add.44, 23 novembre 1994.

161. Observations du Comité des droits de l'homme: Maroc, CCPR/C/79/Add.113, $1^{\mathrm{er}}$ novembre 1999.

162. Observations du Comité des droits de l'homme: Maroc, CCPR/CO/82/MAR (2004), $1^{\text {er }}$ décembre 2004

163. Observations finales du Comité des droits économiques, sociaux et culturels: Maroc, E/C.12/1994/5, 30 mai 1994, \$10.

164. Observations finales du Comité des droits économiques, sociaux et culturels: Maroc, E/C.12/1/Add.55, $1^{\mathrm{er}}$ décembre $2000, \$ 13$.

165. Ibid., $\$ 35$.

166. Observations finales du Comité des droits économiques, sociaux et culturels: Maroc, E/C.12/MAR/CO/2, 4 septembre 2006, $\$ 13 . b$.

167. Ibid., $\$ 35$.

168. A/RES/6o/251, 15 mars 2006

169. Ibid., \$ 4.e. 
Le Maroc a déjà été soumis deux fois à l'examen périodique universel en 2008 et 2012.

Dans l'examen de 2008 le rapport présenté par le Royaume du Maroc ne fait aucune référence à la situation des droits de l'homme au Sahara occidental. On dit seulement que l' " Initiative Marocaine pour la négociation d'un statut d'autonomie de la Région du Sahara » présentée par le Maroc aux Nations unies en 2007 «a intégré les normes et standards internationaux en matière des droits de l'Homme tels qu'ils sont universellement reconnus et consacrés par la Constitution marocaine ${ }^{170}$. Mais cette « initiative» n'est jamais entrée en vigueur. En revanche, le Royaume du Maroc souligne que

[1]e Conseil Royal Consultatif pour les Affaires Sahariennes (CORCAS), institué en 2006, est appelé à émettre des avis consultatifs sur les questions d'ordre général ou spécial se rapportant à la défense de l'intégrité territoriale et de l'unité nationale ainsi qu'au développement humain, économique et social intégré des Provinces du sud $(\text { sic })^{171}$.

Mais on ne connaît jusqu'à aujourd'hui aucune initiative du CORCAS dans le domaine des droits de l'homme. Même s'il est en quelque sorte «normal» de voir le Royaume du Maroc se taire sur la situation des droits de l'homme au Sahara occidental, il est étonnant que le groupe de travail n'ait fait aucune remarque sur cette question ${ }^{172}$. Aucun changement substantiel n'a été opéré concernant ce dernier rapport lors de son approbation par le Conseil ${ }^{173}$.

Lors de l'examen de 2012, la situation a changé. Même si, comme cela était le cas en 2008 , le Maroc n'a pas soulevé la question du respect des droits de l'homme au Sahara occidental dans son rapport (en 2012, on ne parle même plus de l' «initiative» pour la négociation d'un statut d'autonomie) ${ }^{174}$, on constate un changement substantiel parce que dans le dialogue interactif douze pays ont posé des questions au Maroc sur la situation des droits de l'homme au Sahara occidental. De plus, le projet de rapport introduit plusieurs recommandations pour améliorer la situation des droits de l'homme au Sahara occidental occupé ${ }^{175}$. Le rapport définitif introduit quelques petits changements. Parmi les recommandations, certaines ont été appuyées par le Maroc qui considérait que les mesures visées avaient déjà été mises en œuvre ou étaient "en cours d'application » ${ }^{176}$ dont trois sont relatives au Sahara occidental ( $\mathrm{n}^{\circ}$ 130.3, 130.11 et 130.12). On trouve aussi des recommandations qui ne bénéficient pas de l'appui du Maroc dont certaines font référence au Sahara occidental ( $\left.n^{\circ} 131.4\right)$ et figure enfin une recommandation rejetée par le Maroc parce que ce pays a estimé qu'elle ne relevait pas des compétences du Conseil des droits de l'homme ( $\mathrm{n}^{\circ} 132.1$ concernant la création d'une composante droits de l'homme dans la MINURSO).

Il faut remarquer que, lors de l'examen de 2012, l'une des organisations non gouvernementales qui s'était adressée au Conseil des droits de l'homme pour transmettre des observations sur la situation des droits de l'homme au Sahara occidental (Robert F. Kennedy Center for Justice \& Human Rights - RFK Center) a protesté face à plusieurs omissions concernant la question du Sahara occidental décrite dans le rapport du groupe de travail. Le RFK Center remarque cinq omissions relatives à la situation des droits de l'homme au Sahara occidental par les États-Unis et le Danemark, mais également concernant des observations faites par trois pays qui avaient été mentionnés par le rapport (Suède, Costa Rica, Irlande) ${ }^{177}$.

Parmi les procédures spéciales du Conseil des droits de l'homme, on doit faire référence à l'institution de certains rapporteurs spéciaux dont quelques-uns ont présenté des rapports qui traitent de la situation des droits de l'homme au Sahara occidental. Il faut rappeler que depuis la résolution 1979 (2011) du Conseil de sécurité, le Royaume du Maroc s'est engagé à

[...] accorder un accès sans réserves ni restrictions à tous les titulaires de mandats relevant des procédures spéciales du Conseil des droits de l'homme ${ }^{178}$.

Le rapporteur spécial sur la torture et autres peines ou traitements cruels, inhumains ou dégradants (Juan E. Méndez) a présenté le document le plus important ${ }^{179}$. Les références à la situation du Sahara occidental sont nombreuses dans le rapport qui contient un chapitre spécifique sur le Sahara occidental où l'on y trouve des affirmations extrêmement préoccupantes sur la pratique de la torture

170. Rapport national soumis conformément au paragraphe 15(a) de l'annexe à la résolution 5/1 du Conseil des droits de l'homme: Maroc, A/HRC/ WG.6/1/MAR/1, 11 mars 2008, \$9.

171. Ibid., $\$ 44$.

172. Rapport du groupe de travail sur l'examen périodique universel: Maroc, A/HRC/WG.6/1/MAR/4, 10 avril 2008.

173. Rapport du groupe de travail sur l'examen périodique universel: Maroc, A/HRC/8/22, 22 mai 2008.

174. Rapport national présenté conformément au paragraphe 5 de l'annexe à la résolution 16/21 du Conseil des droits de l'homme: Maroc, A/HRC/ WG.6/13/MAR/1, 8 mars 2012.

175. Rapport du groupe de travail sur l'examen périodique universel: Maroc, A/HRC/21/3, 6 juillet 2012. Le rapport informe que dix pays ont évoqué les droits de l'homme au Sahara occidental: Canada ( $\left.n^{\circ} 130.3\right)$, Costa Rica $\left(n^{\circ} 63\right)$, Uruguay $\left(n^{\circ} 132.1\right)$, Espagne $\left(n^{\circ} 130.12\right)$, Suède $\left(n^{\circ} 30\right)$, Norvège $\left(n^{\circ} 131.4\right)$, Autriche ( $\left.n^{\circ} 48\right)$, Ukraine ( $\left.{ }^{\circ} 36\right)$, Royaume-Uni ( $\left.n^{\circ} 38\right)$, Irlande ( ${ }^{\circ} 88$ et 130.11). Deux autres États ont soulevé la question des droits de l'homme au Sahara occidental (États-Unis, Danemark) mais, pour des raisons non exprimées, leurs considérations sur le Sahara occidental ne sont pas mentionnées dans le rapport.

176. Ibid., $\mathrm{n}^{\mathrm{o}} 130$.

177. Robert F. Kennedy Center for Justice \& Human Rights, «Sahrawi Plight Mitigated in UPR Draft Report, Letter to Her Excellency Navanethem Pillay, High Commissioner for Human Rights ", 9 juillet 2012, disponible à l'adresse: http://rfkcenter.org/images/attachments/article/1654/ UPRo70912.pdf.

178. S/RES/1979 du 27 avril 2011, $\$ 12$ du préambule.

179. Rapport du rapporteur spécial sur la torture et autres peines ou traitements cruels, inhumains ou dégradants, Juan E. Méndez. Mission au Maroc, A/HRC/22/53/Add.2, 28 février 2013. Une note préliminaire du rapport indique que le rapporteur a également visité le Sahara occidental (le territoire occupé par le Maroc). 
au Sahara occidental occupé ${ }^{180}$. Les conclusions du rapporteur spécial relevant du Sahara occidental montrent une "pratique ancrée» de la torture contre les personnes qui défendent l'indépendance du Sahara occidental ${ }^{181}$. Parmi les recommandations que fait ce rapporteur spécial au gouvernement marocain, plusieurs sont consacrées au Sahara occidental:

a) D'enquêter rapidement sur toutes les allégations faisant état d'actes de torture et de mauvais traitements pendant et après les manifestations et à la prison de Laâyoune; de tenir les auteurs responsables de ces actes et d'accorder une indemnisation aux victimes;

b) De reconsidérer la compétence du tribunal militaire pour connaître d'affaires concernant des civils dans le cas des 23 Sahraouis en détention à la prison 1 de Salé et de veiller à ce qu'en principe, les civils ne soient pas condamnés par des tribunaux militaires; d'ouvrir des enquêtes sérieuses et impartiales pour établir les faits exacts dans cette affaire et déterminer quelle est la responsabilité des membres de la police ou des forces de sécurité; et d'enquêter sur toutes les allégations de torture et de mauvais traitements;

c) De trouver des moyens pour renforcer encore plus la protection des droits de l'homme internationalement reconnus, notamment en invitant les mécanismes des procédures spéciales de l'Organisation des Nations Unies; en renforçant l'engagement avec la société civile et les institutions nationales des droits de l'homme; et en facilitant la présence des organisations internationales non gouvernementales;

d) La région tout entière tirerait profit de l'établissement d'un mécanisme régional intergouvernemental de surveillance des droits de l'homme, robuste, comme une mesure importante visant à instaurer la confiance et qui peut contribuer à améliorer la situation en ce qui concerne le respect des droits de l'homme et, en particulier, l'interdiction de la torture et autres peines ou traitements cruels, inhumains ou dégradants ${ }^{182}$.

La rapporteuse spéciale dans le domaine des droits culturels (Farida Shaheed) a introduit dans son rapport sur le Maroc un chapitre distinct sur la réalisation des droits culturels au Sahara occidental ${ }^{183}$. D'après ce rapport, la situation des droits culturels au Sahara occidental est aussi très préoccupante ${ }^{184}$. La rapporteuse déclare que:

[s]'agissant du système d'enseignement existant, on a dit que, comme les Sahraouis n'apprenaient que l'histoire officielle du Maroc, rien ne leur était enseigné de leurs propres culture et histoire. L'experte indépendante rappelle que ceci n'est pas conforme à l'article 29 de la Convention relative aux droits de l'enfant et à l'article 5 de la Déclaration de l'UNESCO sur la diversité culturelle ${ }^{185}$.

L'experte indépendante est également préoccupée «par les obstacles importants à l'exercice du droit de participer à la vie culturelle au Sahara occidental». En particulier, «les mines terrestres mettent en péril le style de vie nomade traditionnel, lié au désert, des Sahraouis » et «les organisations non gouvernementales internationales ne sont pas autorisées à participer aux activités de déminage ${ }^{186}$. Une tendance préoccupante a été signalée à l'experte indépendante: «certains Sahraouis ont arrêté de porter leur costume traditionnel distinctif ou hésitent à le faire parce qu'ils se sentent menacés ou harcelés». L'experte indépendante «prie instamment les autorités locales de s'attaquer à ce problème et de prendre des mesures pour promouvoir une culture de tolérance et de diversité culturelle ${ }^{187}$. L'experte indépendante a aussi appris avec préoccupation

[...] que les Sahraouis ne jouissent pas toujours dans la pratique du droit d'enregistrer leurs enfants à l'état civil sous le prénom qu'ils souhaitent, en particulier selon la pratique hassanie des prénoms composés ${ }^{188}$.

En ce qui concerne la conservation du patrimoine culturel immatériel,

l'experte indépendante a été déçue d'apprendre la démolition d'une forteresse espagnole remontant à 1886 et de l'intention des autorités de démolir une église de la même période. Grâce aux efforts de la société civile, l'église n'a pas été totalement démolie et est en train d'être rénovée. La forteresse espagnole a, par contre, été complètement

180. Ibid., $\$ 61-67$, «Laâyoune, Sahara occidental»: «le Rapporteur spécial a trouvé que la torture et les mauvais traitements étaient pratiqués pour extorquer des aveux et que les agents de la force publique faisaient un usage excessif de la force à l'égard des manifestants» (\$62); «Le Rapporteur spécial tient à exprimer sa préoccupation au sujet de l'abandon allégué des victimes aux zones rurales après leur avoir fait subir ces violences» (\$63); «Le Rapporteur spécial a reçu des témoignages crédibles sur la pratique de la torture et des mauvais traitements dans la prison de Laâyoune, notamment les viols, les passages à tabac et la mise à l'isolement pendant plusieurs semaines, en particulier de détenus accusés de participation aux activités en faveur de l'indépendance. [...] Le Rapporteur spécial note avec préoccupation que les allégations de torture et de mauvais traitements pendant la période de presque deux ans qui a précédé les procès [procès d'Akdeim Izik], n'ont fait l'objet d'aucune enquête. Le fait que l'affaire soit devant un tribunal militaire plutôt que civil contribue au manque de transparence et au refus d'enquêter sur les allégations de mauvais traitements» $(\$ 66)$.

181. Ibid., $\$ 72,84-85$.

182. Ibid., $\$ 97$.

183. Rapport de l'experte indépendante dans le domaine des droits culturels, $M^{\text {me }}$ Farida Shaheed. Additif Mission au Maroc, 5-16 septembre 2011, A/HRC/20/26/Add.2, 2 mai 2012. L'experte indépendante s'est aussi rendue à Dakhla, au Sahara occidental, le 14 septembre 2011.

184. Le secrétaire général des Nations unies lui-même a fait une synthèse de ce rapport en indiquant que l'experte indépendante a «recommandé l'abrogation immédiate des mesures qui limitent l'exercice des droits culturels de la population du Sahara occidental, expliquant que plusieurs Sahraouis n'avaient pas été autorisés à donner à leurs enfants des noms hassani» (S/2012/197, \$ 82). Il faut souligner que la visite de l'expert indépendant s'est déroulée après l'adoption de la nouvelle "Constitution" marocaine de 2011.

185. Rapport de l'experte indépendante dans le domaine des droits culturels..., A/HRC/20/26/Add.2, $\$ 71$

186. Ibid., $\$ 72$.

187. Ibid., $\$ 75$.

188. Ibid., $\$ 77$. 
démolie, et a été remplacée par un square. Malheureusement, les pouvoirs publics n'ont pas consulté la population avant de décider de démolir ces bâtiments historiques ${ }^{189}$.

D'après l'experte,

Dans le cadre de l'organisation de nombreux festivals, une partie disproportionnée des fonds a été dépensée pour inviter des musiciens internationaux, originaires en particulier de Mauritanie. Dans le même temps, des formations musicales et musiciens locaux auraient été sélectionnés par un comité habilité à leur demander de modifier, par exemple, les paroles de chansons traditionnelles [...]. Elle a aussi appris que certains d'entre eux n'avaient pas été autorisés à participer aux festivals. De telles pratiques, qui limitent la liberté d'expression de la diversité culturelle, qui devrait pourtant être promue et réalisée, semblent contraires au droit à la liberté d'expression et à la liberté artistique $^{190}$.

La rapporteuse spéciale sur la situation des défenseurs des droits de l'homme (Margaret Sekaggya), dans son rapport présenté en février $2013^{191}$ a examiné quelques plaintes des défenseurs sahraouis des droits de l'homme qui ont présenté des allégations de torture et de mauvais traitements par les forces de sécurité, de refus de réceptionner les documents de constitution de l'Association sahraouie des victimes de graves violations des droits de l'homme (ASVDH) et d'un usage excessif de la force empêchant la tenue de manifestations pacifiques. La rapporteuse

[...] demeure préoccupée par des restrictions relatives à la liberté d'assemblée pacifique, qui représentent un obstacle considérable pour les défenseurs au pays entier, mais surtout pour ceux opérant au Sahara occidental. Un usage excessif de la force durant des manifestations constitue une menace contre l'intégrité physique et mentale des défenseurs. À cet égard, la Rapporteuse spéciale souhaite attirer l'attention du gouvernement marocain sur les dispositions contenues dans la Déclaration sur les défenseurs des droits de l'homme, en particulier l'article 5, alinéa a) ${ }^{192}$.

La Rapporteuse spéciale est aussi préoccupée par des difficultés alléguées d'organisations au Sahara occidental de s'enregistrer auprès des autorités. Dans ce contexte, elle aimerait rappeler au gouvernement du Maroc de l'article 5, alinéas b) et c) de la Déclaration sur les défenseurs des droits de l'homme ${ }^{193}[\ldots]$.
Le groupe de travail sur la discrimination à l'égard des femmes s'est rendu au Maroc et a également souhaité effectuer une visite au Sahara occidental. Le gouvernement marocain lui a proposé d'organiser une visite d'un jour à El-Aaiun ou Dakhla, les deux principales villes du Sahara occidental; mais faute de temps et en raison de contraintes logistiques, le groupe de travail a dû décliner cette proposition. Dans son rapport le groupe affirme que «vu l'intérêt qu'il porte à la situation des femmes dans le territoire non autonome du Sahara occidental, il pourrait envisager de s'y rendre ultérieurement ${ }^{194}$.

Le Maroc a ratifié le 21 juin 1993 la Convention contre la torture et autres peines ou traitements cruels, inhumains ou dégradants (adoptée et ouverte à la signature, à la ratification et à l'adhésion par l'Assemblée générale dans sa résolution 39/46 du 10 décembre 1984). D’après l'article 19.1 de ladite Convention, les États parties présentent au Comité contre la torture, par l'entremise du secrétaire général de l'Organisation des Nations unies, des rapports sur les mesures qu'ils ont prises pour donner effet à leurs engagements en vertu de la Convention, dans un délai d'un an à compter de l'entrée en vigueur de la Convention pour l'État partie intéressé. Les États parties présentent ensuite des rapports complémentaires tous les quatre ans sur toute nouvelle mesure prise, et tout autre rapport demandé par le Comité.

Dans les rapports présentés par le Maroc au Comité en $1994^{195}, 1999^{196}, 2003^{197}$ le Royaume ne fait aucune référence au Sahara occidental. Dans son rapport de 2009, la «région du Sahara» (sic) est seulement mentionnée pour faire allusion au fait que le Maroc a présenté une initiative «d'autonomie» ${ }^{198}$. Le Comité omet également de faire référence au Sahara occidental dans ses observations de $1995^{199}, 1999^{200}$ et $2004^{201}$.

Cependant, les choses ont sensiblement changé. Dans le rapport de $2011^{202}$, le Comité contre la torture a exprimé sa profonde préoccupation face à la pratique de la torture dans le Sahara occidental occupé par le Maroc. Dans ses Observations finales, il consacre à la situation du Sahara occidental plusieurs rubriques et se dit être

[...] préoccupé par les nombreuses allégations de torture et de mauvais traitements commis par les officiers de police, les agents pénitentiaires et plus particulièrement

189. Rapport de l'experte indépendante dans le domaine des droits culturels..., A/HRC/20/26/Add.2, $\$ 79$.

190. Ibid., $\$ 80$.

191. Report of the Special Rapporteur on the Situation of Human Rights Defenders, Margaret Sekaggya, Addendum, A/HRC/22/47/Add.4, 27 février 2013, \$296-302.

192. Ibid., $\$ 300$

193. Ibid., $\$ 301$

194. Rapport du groupe de travail sur l'élimination de la discrimination à l'égard des femmes dans la législation et dans la pratique. Additif Mission au Maroc, A/HRC/20/28/Add.1, 19 juin 2012, \$3.

195. Rapports initiaux des États parties devant être soumis en 1994, Additif, Maroc [29 juillet 1994], CAT/C/24/Add.2, 10 août 1994.

196. Deuxièmes rapports périodiques des États parties devant être présentés en 1998, Additif, Maroc [2 septembre 1998], CAT/C/43/Add.2, 5 janvier 1999.

197. Troisièmes rapports périodiques des États parties devant être présentés en 2002 [23 mars 2003], CAT/C/66/Add.1 et Corr.1, 21 mai 2003.

198. Quatrièmes rapports périodiques des États parties devant être soumis en 2006, Maroc [27 avril 2009], CAT/C/MAR/4, 5 novembre 2009, $\$ 5$.

199. Rapport du Comité contre la torture. Assemblée générale. Documents officiels. Cinquantième session, supplément no 44, A/50/44 SUPP.

200. Rapport du Comité contre la torture. Assemblée générale. Documents officiels. Cinquante-quatrième session, supplément $n^{\circ} 44, \mathrm{~A} / 54 / 44 \mathrm{SUPP}$.

201. Conclusions et recommandations du Comité contre la torture: Maroc, CAT/C/CR/31/2, 5 février 2004.

202. Observations finales du Comité contre la torture: Maroc, CAT/C/MAR/CO/4, 21 décembre 2011. 
les agents de la Direction de surveillance du territoire (DST) - désormais reconnus comme officiers de police judiciaire - lorsque les personnes sont privées de l'exercice des garanties juridiques fondamentales comme l'accès à un avocat, en particulier celles suspectées d'appartenir à des réseaux terroristes ou d'être des partisans de l'indépendance du Sahara occidental ou durant les interrogatoires dans le but de soutirer des aveux aux personnes suspectées de terrorisme (art. 2, 4, 11 et 15 de la Convention) ${ }^{203}$.

Le Comité consacre deux amples rubriques aux «Événements concernant le Sahara occidental» et au «Camp Gdeim Izik».

Sur la première question, il

[...] est préoccupé par les allégations reçues sur la situation au Sahara occidental, où seraient pratiquées des arrestations et des détentions arbitraires, des détentions au secret et dans des lieux secrets, des tortures, des mauvais traitements, des extorsions d'aveux sous la torture et un usage excessif de la force par les forces de sécurité et par les forces de l'ordre marocaines. Le comité rappelle encore une fois qu'en vertu de la Convention contre la torture et autres peines ou traitements cruels, inhumains ou dégradants aucune circonstance exceptionnelle quelle qu'elle soit ne saurait être invoquée pour justifier la torture sur le territoire soumis à la juridiction de l'État partie et que les mesures de maintien de l'ordre ainsi que les procédures d'enquête et d'investigation doivent être appliquées dans le plein respect du droit international relatif aux droits de l'homme, ainsi que des procédures judiciaires et des garanties fondamentales en vigueur dans l'État partie. L'État partie devrait prendre d'urgence des mesures concrètes pour prévenir les actes de torture et les mauvais traitements décrits précédemment. En outre, il devrait annoncer une politique de nature à produire des résultats mesurables par rapport à l'objectif d'éliminer tout acte de torture et tout mauvais traitement de la part des agents de l'État. L'État partie devrait renforcer les mesures prises pour que des enquêtes approfondies, impartiales et efficaces soient menées rapidement sur toutes les allégations de torture et de mauvais traitement infligés à des prisonniers, à des détenus et sur tous les autres $\operatorname{cas}^{204}$.

Sur la deuxième question, le Comité est « tout particulièrement préoccupé» par les circonstances qui ont entouré l'évacuation du camp de Gdeim Izik en novembre 2010. Au cours de cette évacuation, plusieurs personnes ont été tuées, y compris des agents des forces de l'ordre, et des centaines d'autres arrêtées. Le Comité reconnaît que la grande majorité des personnes arrêtées ont été depuis remises en liberté dans l'attente de leur procès. Cependant, «il reste sérieusement préoccupé par le fait que lesdits procès se dérouleront devant des tribunaux militaires alors que les intéressés sont des civils». De plus, le Comité se déclare préoccupé par le fait
[...] qu'aucune enquête impartiale et efficace n'ait été ouverte pour faire la lumière sur ces événements et établir les responsabilités éventuelles au sein des forces de l'ordre (art. 2, 11, 12, 15 et 16).

\section{Le Comité observe que l'État partie}

[...] devrait renforcer les mesures prises pour que des enquêtes approfondies, impartiales et efficaces soient menées rapidement sur les violences et les décès survenus à l'occasion du démantèlement du camp de Gdeim Izik, et que les responsables soient traduits en justice. L'État partie devrait modifier sa législation afin de garantir à toutes les personnes civiles d'être jugées exclusivement par des juridictions civiles ${ }^{205}$.

Enfin, les observations du Comité sont tout particulièrement critiques envers les travaux de l'IER concernant le Sahara occidental. Dans le document, le Comité dit rester «toutefois préoccupé» par le fait que les travaux de l'IER «ne sont pas complets puisqu'ils n'incluent pas les violations commises au Sahara occidental» et que «certains cas de disparitions forcées n'étaient pas résolus à la fin des travaux de l'IER en 2005». De plus, le Comité

[...] est préoccupé par le fait que les travaux de l'IER puissent avoir entraîné une impunité de facto des auteurs des violations de la Convention commises au cours de cette période, puisqu'à ce jour aucun d'entre eux n'a été poursuivi. Enfin, le Comité est préoccupé par les informations reçues selon lesquelles toutes les victimes et toutes les familles de victimes n'auraient pas été indemnisées et que les indemnités versées n'auraient pas toujours été équitables, adéquates ou effectives (art. 12, 13 et 14).

Selon le Comité, l'État partie devrait donc

[...] s'assurer que le Conseil national des droits de l'homme, qui a été désigné pour finaliser les travaux de l'IER, continue de s'efforcer d'élucider les cas de disparitions forcées intervenues entre 1956 et 1999 restés non élucidés, y compris les cas liés au Sahara occidental.

\section{Le Comité ajoute que}

[1]'État partie devrait également intensifier ses efforts pour assurer aux victimes de torture et de mauvais traitements une réparation sous la forme d'une indemnisation équitable et suffisante et d'une réadaptation aussi complète que possible. À cet effet, il devrait inclure dans sa législation des dispositions sur le droit des personnes victimes de torture d'être indemnisées de manière équitable et adéquate du préjudice ainsi subi ${ }^{206}$.

\section{L'éventualité d'une Cour pénale internationale}

Le Maroc a reconnu officiellement, bien que partiellement, l'existence des violations graves des droits de l'homme 
jusqu'en 1999. Mais plusieurs institutions internationales, États et organisations non gouvernementales, ont dénoncé la persistance des violations des droits de l'homme depuis 1999 jusqu'à la période actuelle, même si le type des droits non respectés a connu quelques changements. Devant l'absence d'un mécanisme international de vigilance, et après avoir constaté que les violations des droits de l'homme restent impunies dans le cadre de l'appareil institutionnel marocain, on a suggéré la possibilité d'effectuer une pression internationale forte avec l'instauration d'une cour pénale internationale. À ce sujet deux possibilités ont été étudiées. La première est d'instituer une cour pénale internationale ad hoc suivant quelques précédents (Yougoslavie, Rwanda, Liban), mais il faudrait alors approuver une résolution dans le cadre du chapitre VII de la Charte des Nations unies, ce qui semble très improbable. La seconde possibilité est d'essayer de saisir la Cour pénale internationale déjà existante malgré le fait que le Maroc ait refusé de ratifier le traité qui établit cette Cour. On a suggéré trois voies pour surmonter cet obstacle. La première est que le Maroc peut manifester une acceptation ad hoc de la juridiction du tribunal, d'après l'article 12(3) du Statut de Rome. Mais il est difficile de croire que les autorités qui ne veulent pas poursuivre les crimes voudraient autoriser une institution internationale à le faire. La deuxième est que le Conseil de sécurité défère une affaire à la Cour, mais encore faut-il le faire suivant le chapitre VII de la Charte des Nations unies. La troisième est que la République sahraouie puisse être reconnue comme un État membre des Nations unies (comme la Palestine) et ratifie le Statut de Rome, pour autodéférer l'affaire à la Cour pénale, avec la possibilité d'élargir la juridiction de la Cour jusqu'en $2002^{207}$.

Cette argumentation n'épuise pas les possibilités d'établir une justice internationale. En Sierra Leone, a ainsi été établie une cour internationale par une résolution du Conseil de sécurité suivant le chapitre VI de la Charte ${ }^{208}$, avec un accord préalable entre les Nations unies et le Sierra Leone qui présente de faibles probabilités de se reproduire avec le Maroc. Au Cambodge la cour internationale a été créée par une résolution de l'Assemblée générale des Nations unies ${ }^{209}$, mais également avec un accord préalable entre les Nations unies et le Cambodge. Le modèle le plus proche du Sahara occidental est peut-être à rechercher au Timor oriental, où l'Administration transitoire des Nations unies a créé des cours spéciales pour juger les violations des droits de l'homme ${ }^{210}$.

207. Voir H. Sántha, Y. Lennartson Hartmann, M. Klamberg, "Crimes Against Humanity in Western Sahara: The Case Against Morocco », Juridisk Publikation, vol. 2, 2010, p. 196-197.

208. S/RES/1315, 14 août 2000.

209. A/RES/57/228 A, 27 février 2003 et A/RES/57/228 B, 22 mai 2003.

210. L'Administration transitoire des Nations unies au Timor oriental (ATNUTO), fut établie par la résolution 1272 du Conseil de sécurité (S/RES/1272) du 25 octobre 1999. 